\title{
MODELAGEM HIPSOMÉTRICA DE POVOAMENTOS DE ACÁCIA- NEGRA USANDO LINGUAGEM DE PROGRAMAÇÃO JULIA
}

\author{
HIPSOMETRIC MODELING OF BLACK WATTLE STANDS USING JULIA LANGUAGE
}

\author{
João Felipe Cardozo Martins', Dimas Agostinho Silva', Alexandre Behling ${ }^{1}$, Richardson Ribeiro ${ }^{2}$ \\ ${ }^{1}$ Universidade Federal do Paraná, Curitiba, Paraná, Brasil - jmartins.ufpr@gmail.com, \\ dimas.agostinho.silva@gmail.com \& alexandre.behling@gmail.com \\ ${ }^{2}$ Universidade Tecnológica do Paraná, Pato Branco, Paraná, Brasil - prof.richard@gmail.com
}

\begin{abstract}
RESUMO
A modelagem hipsométrica para indivíduos florestais é recorrente na área florestal, havendo necessidade de reafirmar-se métodos e técnicas para mensurar altura e buscar melhores informações para as variáveis dendrométricas. A espécie acácia-negra (Acacia mearsii De Wild.) é reconhecida no sul do Brasil como uma espécie de alto valor comercial, apresentando a quarta maior área plantada de povoamentos florestais no Rio Grande do Sul, dessa forma, estudos a respeito da espécie são de grande importância para o setor florestal. O presente trabalho visa à obtenção de estimativas hipsométricas individuais para árvores de acácia negra, em que (onde) foram propostos e ajustados 50 modelos hipsométricos, usando a Linguagem de Programação Julia. Empregou-se dados de árvores de acácia-negra com idades entre 1 e 10,75 anos, provenientes de um experimento nas regiões de Cristal, Encruzilhada do Sul e Piratini, estado do Rio Grande do Sul. Os dados foram divididos em 4 grupos, de acordo com sua fase de crescimento (Jovem, Média-Inicial, Média-Avançada e Madura). Utilizou-se de 550 árvores para o ajuste de modelos, além de 30 indivíduos de cada grupo, previamente separados de maneira aleatória, para a validação do modelo de melhor desempenho. Avaliou-se os ajustes pelos seguintes critérios: Viés, Precisão, Acurácia e gráficos de dispersão dos resíduos. O modelo Parabólico foi o que melhor se ajustou aos dados nas classes Jovem, e o modelo Exponencial para as demais classes de idades foram os que apresentaram os melhores desempenhos. Sua validação apresentou resultados satisfatórios para o erro padrão da estimativa e para o teste de Qui-quadrado, teve valores de 2,0239, $1,0364,118,1416$ e 0,6158 . Sendo assim, observou-se que os modelos são aptos para uso em outras bases de dados com exceção da classe Média-Avançada e que a linguagem de programação Julia é uma alternativa para processos e softwares de linguagem de programação já consolidados na área florestal.
\end{abstract}

PALAVRAS-CHAVE: Altura total, Modelos hipsométricos, Plantios florestais, Rio Grande do Sul.

\section{ABSTRACT}

Hypsometric modeling for forest individuals is recurrent in the forest area, with the need to reaffirm methods and techniques to measure height and seek better information for dendrometric variables. The black wattle species (Acacia mearsii De Wild.) is recognized in southern Brazil as a species of high commercial value, presenting the fourth largest planted area of forest stands in Rio Grande do Sul, thus, studies on the species are of great importance for the forestry sector. The present work aims to obtain individual hypsometric estimates for black wattle trees, in which (where) 50 hypsometric models were proposed and adjusted, using the Julia Programming Language. Data from black wattle trees aged between 1 and 10.75 years, from an experiment in the regions of Cristal, Encruzilhada do Sul and Piratini, state of Rio Grande do Sul, were used. According to their stage of growth (Young, Middle-Initial, MiddleAdvanced and Mature). 550 trees were used to adjust models, in addition to 30 individuals from each group, previously randomly separated, to validate the best performing model. The adjustments were evaluated by the following criteria: Bias, Precision, Accuracy, and residual scatter plots. The Parabolic model was the one that best fit the data in the young classes, and the Exponential model for the other age classes showed the best performance. Its validation presented satisfactory results for the standard error of the estimate and for the Chi-square test, it had values of 2.0239, 1.0364, 118.1416 and 0.6158 . Thus, it was observed that the models are suitable for use in other databases except for the Middle-Advanced class and that the Julia programming language is an alternative for programming language software and processes already consolidated in the forestry area.

KEYWORDS: Total height, Hypsometric models, Forest plantations, Rio Grande do Sul. 


\section{INTRODUÇÃO}

As plantações florestais no Brasil atingiram 9 milhões de hectares em 2019, contudo, apenas 5\% desta área são ocupadas por outras espécies que não sejam pinus e eucalipto, como por exemplo a acácia-negra, que corresponde a quarta espécie em área plantada no país (IBÁ, 2020). De origem australiana, a Acacia mearnsii De Wild, pertencente à família Fabaceae e popularmente conhecida como acácia-negra. No Brasil a maior concentração dos plantios comerciais dessa espécie está localizada no estado do Rio Grande do Sul, onde, segundo Vieira \& Schumacher (2010) a madeira é utilizada para a produção de celulose e carvão e da casca é extraído o tanino, empregado principalmente na indústria farmacêutica e coureira.

Os atributos comumente avaliados na dinâmica florestal são: o crescimento, a mortalidade e o recrutamento (BARRETO et al., 2016). As estimativas para esses atributos são obtidas, principalmente, a partir de inventários florestais contínuos, com base em parcelas permanentes, monitoradas a médio e longo prazos (FIGUEIREDO et al., 2010). O crescimento da vegetação está relacionado a disponibilidade de recursos naturais e a capacidade de cada espécie em utilizá-los (ABRÃO et al., 2017).

O conjunto de alturas das árvores medidas, com os respectivos diâmetros, é usado para estabelecer uma relação de regressão da altura em função do diâmetro (modelo de relação hipsométrica), a qual é empregada para estimar as alturas das demais árvores da parcela em função dos diâmetros já medidos. Logo, estima-se a variável de difícil acesso em função da mais fácil e de rápida medição, reduzindo-se, portanto, o tempo e os custos de mensuração (BARTOSZECK et al., 2004).

A medição de alturas das árvores em um povoamento florestal é considerada uma atividade onerosa, sendo usual medir a altura de apenas algumas árvores da parcela durante o inventário florestal (STOLLE et al., 2018). Machado \& Figueiredo Filho (2009) afirmam que a altura é a segunda variável independente utilizada nos métodos estimativos em funções de afilamento e em outras relações dendrométricas. Nos inventários florestais, as alturas das árvores não medidas dentro da parcela são estimadas por meio de uma relação hipsométrica expressa por uma equação ajustada (MACHADO \& FIGUEIREDO FILHO, 2009; SANQUETTA et al., 2014a).

A relação hipsométrica não apresenta um relacionamento biológico bem definido, tal como altura e idade ou diâmetro e idade, havendo grande variabilidade em altura para um mesmo diâmetro em sítio e idades diferentes (CHAPMAN \& MEYER, 1949; AZEVEDO, 1999).

A dificuldade da escolha de um modelo para representar essas relações hipsométricas se deve à não linearidade da relação entre as variáveis envolvidas e as restrições impostas aos parâmetros dos modelos, por razões biológicas (PLÁCIDO et al., 2004; SOARES et al., 2004). Esses dois fatores aliados tornam o ajuste de modelos estatísticos para representar as relações hipsométricas um problema de regressão não linear, com restrições nos parâmetros.

Nesse contexto, considerando que a cubagem rigorosa é um processo de alto custo e que o ajuste de equações hipsométrica auxilia na predição da altura de indivíduos de acácia-negra, pretende-se com este trabalho:

a. Ajustar os modelos hipsométricos.

b. Comparar os modelos hipsométricos por meio das estatísticas: Viés, Precisão e Acurácia;

c. Avaliar os resíduos para o melhor modelo;

d. Avaliar os resultados estatísticos obtidos com os dados de validação.

e. Processar os dados e validar os resultados utilizando a linguagem de programação Julia.

\section{MATERIAL E MÉTODOS}

\section{Origem dos dados}

As áreas estudadas localizam-se no estado do Rio Grande do Sul e são provenientes das regiões de Cristal, Encruzilhada do Sul e Piratini. Segundo a classificação climática de Köppen-Geiger as três regiões possuem clima quente e temperado ( $\mathrm{Cfa}$ ).

Os povoamentos estudados tiveram variação entre 1 e 10,75 anos. A amostragem abrangeu doze povoamentos, de forma que em cada um foram instaladas, aleatoriamente, quatro parcelas circulares de $400 \mathrm{~m}^{2}$, onde no interior de cada uma foi posicionada uma subparcela de 78,54 $\mathrm{m}^{2}$ com diâmetro de $5 \mathrm{~m}$, a fim de viabilizar a coleta de variáveis de alto custo (biomassa $e$ teores de carbono da biomassa), dessa forma foi amostrada a biomassa do fuste e copa de 670 árvores. Os dados foram agrupados qualitativamente de acordo com a idade dos povoamentos, formando quatro classes conforme observado na Tabela 1.

Os autores optaram por considerar que a maior divisão dos estratos não deveria incluir tamanho de amplitude maior que dois anos, porque, dessa maneira, não seriam evidenciados os efeitos de tais proporções. 
Tabela 1. Idade qualitativa dos povoamentos de acácianegra amostrados no estado do Rio Grande do Sul, Brasil.

\begin{tabular}{ccc}
\hline Local & Idade (anos) & Classe \\
\hline \multirow{3}{*}{ Cristal } & 1,75 & Jovem \\
& 2,75 & Média Inicial \\
\cline { 2 - 3 } & 5,00 & Média Avançada \\
& 10,08 & Madura \\
\hline \multirow{3}{*}{ Encruzilhada } & 1,83 & Jovem \\
& 3,08 & Média Inicial \\
& 5,75 & Média Avançada \\
\hline \multirow{3}{*}{ Piratini } & 10,75 & Madura \\
& 1,00 & Jovem \\
& 2,33 & Média Inicial \\
& 5,25 & Média Avançada \\
\hline
\end{tabular}

\section{Estatística descritiva}

Para caracterizar os povoamentos de acácia-negra utilizados no ajuste dos modelos, utilizaram-se certos elementos estatísticos, como: média $(\bar{x})$, variância $\left(S_{x}{ }^{2}\right)$, desvio padrão $(S)$, erro padrão $\left(S_{\bar{x}}\right)$ e o intervalo de confiança $(I C)$ para as variáveis dendrométricas: $d$ e $h$.

\section{Modelos}

Foram ajustados 51 modelos hipsométricos, conforme Tabela 2.

Tabela 2. Modelos hipsométricos testados para o do povoamento de Acacia mearnsii em povoamentos no Rio Grande do Sul.

\begin{tabular}{cc}
\hline Autor & Modelo \\
\hline Assmann (1) & $h=\beta_{0}+\beta_{1} \cdot\left(\frac{1}{d}\right)$ \\
\hline Assmann (2) & $h=\beta_{0}+\beta_{1} \cdot\left(\frac{1}{d^{2}}\right)$ \\
\hline Azevedo et al. (1999) (1) & $\frac{h}{\ln (d)}=\beta_{0}+\beta_{1} \cdot d^{2}$ \\
\hline Azevedo et al. (1999) (2) & $\ln (h)=\beta_{0}+\beta_{1} \cdot d^{2}$ \\
\hline Azevedo et al. (1999) (3) & $\frac{d}{\sqrt{h}}=\beta_{0}+\beta_{1} \cdot d$ \\
\hline Azevedo et al. (1999) (4) & $\sqrt{h}=\beta_{0}+\beta_{1} \cdot \frac{1}{d}+\beta_{2} \cdot d^{2}$ \\
\hline Azevedo et al. (1999) (5) & $h=\beta_{0}+\beta_{1} \cdot d^{2}$ \\
\hline Azevedo et al. (2011) (1) & $\frac{d}{\sqrt{h}}=\beta_{0}+\beta_{1} \cdot d^{2}$ \\
\hline Azevedo et al. (2011) (2) & $\frac{d}{\sqrt{h}}=\beta_{0}+\beta_{1} \cdot \frac{1}{d}+\beta_{2} \cdot d^{2}$ \\
\hline Azevedo et al. (2011) (3) & $h=\beta_{0}+\beta_{1} \cdot \frac{1}{d}+\beta_{2} \cdot d^{2}$ \\
\hline Azevedo et al. (2011) (4) & $\ln (h)=\beta_{0}+\beta_{1} \cdot \frac{1}{d}+\beta_{2} \cdot d^{2}$ \\
\hline Azevedo et al. (2011) (5) & $\sqrt{h}=\beta_{0}+\beta_{1} \cdot \sqrt{d}$ \\
\hline Azevedo et al. (2011) (6) & $\sqrt{h}=\beta_{0}+\beta_{1} \cdot \ln (d)$ \\
\hline
\end{tabular}

\begin{tabular}{|c|c|}
\hline Autor & Modelo \\
\hline Backman modificada & $h=\beta_{0}+\beta_{1} \cdot \ln (d)+\beta_{2} \cdot \ln ^{2}(d)$ \\
\hline Berkhout & $h=\beta_{0}+d^{\beta_{1}}$ \\
\hline Curtis (1967) & $\ln (h)=\beta_{0}+\beta_{1} \cdot\left(\frac{1}{d}\right)$ \\
\hline Exponencial (1) & $\ln (h)=\beta_{0}+\beta_{1} \cdot \frac{1}{d}+\beta_{2} \cdot i$ \\
\hline Exponencial (2) & $\mathrm{h}=\beta_{0} \cdot e^{\beta_{1} \cdot d}$ \\
\hline Exponencial (3) & $\mathrm{h}=\beta_{0} \cdot e^{\beta_{1} \cdot d^{2}}$ \\
\hline Exponencial (4) & $\mathrm{h}=\beta_{0} \cdot e^{\beta_{1} \cdot \frac{1}{d}}$ \\
\hline Finger (1992) & $h=\beta_{0}+\beta_{1} \cdot\left(\frac{1}{d^{2}}\right)$ \\
\hline Henricksen (1950) & $h=\beta_{0}+\beta_{1} \cdot \ln (d)$ \\
\hline Hiperbólico (1) & $h=\beta_{0}+\beta_{1} \cdot \frac{1}{d^{2}}+\beta_{2} \cdot i$ \\
\hline Linear Simples & $h=\beta_{0}+\beta_{1} \cdot d$ \\
\hline Naslung (1) & $h-1,30=\frac{d}{\beta_{0}+\beta_{1} \cdot d^{2}}$ \\
\hline Naslung (2) & $\frac{d}{\sqrt{h-1,30}}=\beta_{0}+\beta_{1} \cdot d$ \\
\hline Naslung (Prodan) (1) & $h-1,30=\frac{d}{\beta_{0}+\beta_{1} \cdot d+\beta_{2} \cdot d^{2}}$ \\
\hline Naslung (Prodan) (2) & $h=\frac{d}{\beta_{0}+\beta_{1} \cdot d+\beta_{2} \cdot d^{2}}$ \\
\hline Não Referenciado & $\frac{1}{\sqrt{h}}=\beta_{0}+\beta_{1} \cdot\left(\frac{1}{d}\right)$ \\
\hline Parabólico (1) & $h=\beta_{0}+\beta_{1} \cdot d+\beta_{2} \cdot d^{2}$ \\
\hline Parabólico (2) & $h=\beta_{0}+\beta_{1} \cdot d+\beta_{2} \cdot d^{2}+\beta_{3} \cdot i$ \\
\hline Parabólico (3) & $\ln (h)=\beta_{0}+\beta_{1} \cdot d+\beta_{2} \cdot d^{2}$ \\
\hline Petterson (1) & $\frac{1}{\sqrt[3]{h-1,30}}=\beta_{0}+\beta_{1} \cdot\left(\frac{1}{d}\right)$ \\
\hline Petterson (2) & $\frac{1}{\sqrt{h}}=\beta_{0}+\beta_{1} \cdot\left(\frac{1}{d}\right)$ \\
\hline Pollanschutz & $\ln (h-1,30)=\beta_{0}+\beta_{1} \cdot\left(\frac{1}{d}\right)$ \\
\hline Potencial (1) & $\ln (h)=\beta_{0}+\beta_{1} \cdot \ln (d)+\beta_{2} \cdot i$ \\
\hline Potencial (2) & $h=\beta_{0}+\left(d^{2}\right)^{\beta_{1}}$ \\
\hline Potencial (3) & $h=\beta_{0}+\left(\frac{1}{d}\right)^{\beta_{1}}$ \\
\hline Prodan (1965) (1) & $\frac{d^{2}}{h}=\beta_{0}+\beta_{1} \cdot d+\beta_{2} \cdot d^{2}$ \\
\hline Prodan (1965) (2) & $\frac{d^{2}}{h-1,30}=\beta_{0}+\beta_{1} \cdot d+\beta_{2} \cdot d^{2}$ \\
\hline Sanquetta et al. (2013) & $h=\beta_{0}+\beta_{1} \cdot \frac{1}{d}+\beta_{2} \cdot d$ \\
\hline Schneider (1986) (1) & $h=\beta_{0}+\beta_{1} \cdot \frac{1}{d}$ \\
\hline Schneider (1986) (2) & $h-1,30=\beta_{0}+\beta_{1} \cdot d$ \\
\hline Schumacher (1986) (1) & $h-1,30=\beta_{0}+\beta_{1} \cdot d+\beta_{2} \cdot d^{2}$ \\
\hline Schumacher (1986) (2) & $\ln (h)=\beta_{0}+\beta_{1} \cdot \ln (d)+\beta_{2} \cdot i$ \\
\hline Schumacher-Hall & $\ln (h)=\beta_{0}+\beta_{1} \cdot \ln (d)+\beta_{2} \cdot \ln (i)$ \\
\hline Semilogarítmico & $h=\beta_{0}+\beta_{1} \cdot \ln (d)+\beta_{2} \cdot i$ \\
\hline Silva (1980) & $\ln (h)=\beta_{0}+\beta_{1} \cdot \ln (d)+\beta_{2} \cdot\left(\frac{1}{d}\right)$ \\
\hline Stoffels \& Soest (1953) (1) & $\ln (h)=\beta_{0}+\beta_{1} \cdot \ln (d)$ \\
\hline Stoffels \& Soest (1953) (2) & $\ln (h)=\beta_{0}+\beta_{1} \cdot \log (d)$ \\
\hline Trorey (1932) & $h=\beta_{0}+\beta_{1} \cdot d+\beta_{2} \cdot d^{2}$ \\
\hline
\end{tabular}

Em que: $\mathrm{d}=$ diâmetro altura do peito $(\mathrm{cm}) ; \mathrm{h}=$ altura total $(\mathrm{m})$; $\ln =$ logaritmo neperiano; $\beta_{0}, \beta_{1}, \beta_{2}$ e $\beta_{3}=$ parâmetros. 


\section{Seleção da melhor equação}

Para avaliar a qualidade preditiva dos modelos de altura optou-se pelo uso das estatísticas de viés (1.1 e 1.2), precisão (2.1 e 2.2) e acurácia (3.1 e 3.2).

$$
\begin{gathered}
\bar{e}=\frac{\sum_{i=1}^{n} e_{i}}{n}=\frac{\sum_{i=1}^{n}\left(h-h_{e s t}\right)}{n} \\
\bar{e}_{\%}=\frac{\bar{e}}{\bar{h}} 100 \\
S_{e}=\sqrt{\frac{\sum_{i=1}^{n}\left(h-\bar{e}-h_{e s t}\right)^{2}}{n-1}}=\sqrt{\frac{\sum_{i=1}^{n}\left(e_{i}-\bar{e}\right)^{2}}{n-1}} \\
S_{e_{\%}}=\frac{S_{e}}{h} 100 \\
m_{x}=\sqrt{\left(S_{e}\right)^{2}+(\bar{e})^{2}} \\
m_{x_{\%}}=\frac{m_{x}}{\bar{h}} 100
\end{gathered}
$$

Em que: $\bar{e}$ é o viés absoluto, $\bar{e}_{\%}$ é o viés relativo, $e_{i}$ é o erro sistemático para i-ésima árvore, $h$ é a altura observada, $h_{e s t}$ é a altura estimada, $\bar{b}$ é a média da biomassa observada, $n$ é o número de árvores, $S_{e}$ é a precisão absoluta, $S_{e_{\%}}$ é a precisão relativa, $m_{x}$ é a acurácia absoluta e $m_{x_{\%}}$ é a acurácia relativa.

\section{Validação dos dados}

Ao se avaliar as equações hipsométricas apresentadas são apropriadas para outros indivíduos não inclusos no processo de modelagem, utilizou-se 30 indivíduos da mesma classe de idade, optou-se por esse valor para padronizar e para manter uma quantidade de até $20 \%$ de cada classe, para que o número de indivíduos arbóreos seja representativo. Dessa forma, calculou-se o Quiquadrado $\left(\chi^{2}\right)(4)$, com nível de significância $(\alpha)$ de 0,05.

$$
\chi^{2}=\sum_{i=1}^{n} \frac{\left(y_{i}-\hat{y}_{i}\right)^{2}}{y_{i}}
$$

Em que: $y_{i}=$ altura real para cada observação; $\hat{y}_{i}=$ altura estimada para cada observação.

\section{Software}

As análises estatísticas foram realizadas utilizando a linguagem de programação científica denominada Julia, na versão 1.5.3, utilizando a plataforma Visual Studio Code. Mais detalhes dessa linguagem de programação podem ser encontrados em https://julialang.org/.

\section{Programação}

\begin{tabular}{|c|c|}
\hline julia> & using Pkg \\
\hline Julia> & Pkg.add("CSV") \\
\hline julia> & Pkg.add("Statistics") \\
\hline julia> & Pkg.add(“Distributions") \\
\hline julia> & Pkg.add(“DataFrames") \\
\hline julia> & Pkg.add(“XLSX”) \\
\hline julia> & Pkg.add(“PyPlot") \\
\hline Julia> & $\begin{array}{l}\text { using CSV, Statistics, Distributions, DataFrames, } \\
\text { XLSX, PyPlot }\end{array}$ \\
\hline julia> & $\begin{array}{l}\text { dados=CSV.read("C://Usuário//Pasta1//Pasta2// } \\
\text { PastaN//dados.csv") }\end{array}$ \\
\hline
\end{tabular}

Para criação dos modelos foram utilizados os seguintes pacotes: CSV, Statistics, Distributions, DataFrames, XLSX e
PyPlot. No Algoritmo 1 tem-se os comandos para carregamento de pacotes e da base de dados.

\begin{tabular}{|c|c|}
\hline julia> & matrix_um = ones(length(dados.d),1); \\
\hline julia> & $\mathrm{x} 1=[$ matrix_um dados.d]; \\
\hline julia> & $b 1=\operatorname{inv}\left(x 1^{\prime} \times 1\right)^{*} \times 1^{\prime *}($ dados.h $)$ \\
\hline julia> & Linear $=b 1[1] .+b 1[2] .{ }^{*}$ dados.d; \\
\hline julia> & $x 2=[$ matrix_um $($ dados.d).^2] \\
\hline julia> & b2 $=\operatorname{inv}\left(\times 2^{\prime} \times 2\right)^{*} \times 2^{\prime *}(($ dados.h $) . /(\log .($ dados.d $)))$ \\
\hline \multirow[t]{2}{*}{ julia> } & Azevedo_1999_1 \\
\hline & b2[2].*((dados.d).^2)).*(log.(dados.d)); \\
\hline julia> & x3 = [matrix_um (log.(dados.d))]; \\
\hline julia> & b3 $=\operatorname{inv}\left(x 3^{\prime} \times 3\right)^{*} \times 3^{\prime *}($ dados.h $)$ \\
\hline julia> & Henricksen $=b 3[1] .+b 3[2] . *(\log .($ dados.d $))$ \\
\hline julia> & x4 = [matrix_um (1./(dados.d))]; \\
\hline julia> & b4 $=\operatorname{inv}\left(x 4^{\prime} \times 4\right)^{*} \times 4^{\prime *}(\log .($ dados.h $)) ;$ \\
\hline julia> & Curtis $=(b 4[1] .+b 4[2] . *(1 . /($ dados.d $))) ;$ \\
\hline julia> & sse_Curtis $=\operatorname{sum}\left((\right.$ Curtis- $(\log .($ dados.h $\left.))) \cdot{ }^{\wedge} 2\right)$; \\
\hline julia> & $\begin{array}{l}\text { Syx_Curtis = sqrt((sse_Curtis./(length((dados.h))- } \\
2))) ;\end{array}$ \\
\hline julia> & FCM_Curtis = exp.((Syx_Curtis.^2). $\left.{ }^{*} 0.5\right)$ \\
\hline julia> & $\begin{array}{l}\text { Curtis }=\text { exp.(b4[1] } .+\quad b 4[2] . *(1 \quad . / \\
(\text { dados.d))).*FCM_Curtis; }\end{array}$ \\
\hline julia> & x5 = [matrix_um dados.d (dados.d).^2]; \\
\hline julia> & b5 = inv $\left(\times 5^{\prime} \times 5\right)^{*} \times 5^{\prime *}($ dados. $h)$ \\
\hline \multirow[t]{2}{*}{ julia> } & Trorey \\
\hline & $b 5[1] .+\left(b 5[2] .{ }^{*}\right.$ dados.d $) .+(b 5[3] . *(($ dados.d $) . \wedge 2))$ \\
\hline julia> & x6 $=[$ matrix_um (log.(dados.d)) $]$ \\
\hline julia> & $b 6=\operatorname{inv}\left(\times 6{ }^{\prime} \times 6\right)^{*} \times 6^{\prime *}(\log .($ dados.h $)) ;$ \\
\hline julia> & Stoffels $=(b 6[1] .+(b 6[2] . *(\log .($ dados.d $)))) ;$ \\
\hline julia> & sse_Stoffels $=\operatorname{sum}\left((\right.$ Stoffels-(log. $($ dados.h) $\left.)) \cdot^{\wedge} 2\right)$ \\
\hline \multirow[t]{2}{*}{ julia> } & Syx_Stoffels \\
\hline & sqrt((sse_Stoffels./(length((dados.h))-2))); \\
\hline julia> & FCM_Stoffels $=\exp .\left(\left(S y x \_S t o f f e l s . \wedge 2\right) *^{*} 0.5\right)$ \\
\hline \multirow[t]{2}{*}{ julia> } & Stoffels \\
\hline & $\begin{array}{l}\text { exp.(b6[1].+(b6[2].*(log.(dados.d)))).*FCM_Stoff } \\
\text { els; }\end{array}$ \\
\hline
\end{tabular}

Algoritmo 1. Instalação e carregamento dos pacotes e da base de dados.

No Algoritmo 2 observa-se todos os comandos para elaborar os modelos hipsométricos.

Algoritmo 2. Elaboração dos modelos hipsométricos. 


\begin{tabular}{|c|c|}
\hline $\begin{array}{l}\text { julia> } \\
\text { julia> }\end{array}$ & $\begin{array}{l}x 7=[\text { matrix_um dados.d (dados.d).^2 dados.i }] \\
b 7=\operatorname{inv}\left(x 7^{\prime} x 7\right)^{*} x 7^{\prime *}(\text { dados.h })\end{array}$ \\
\hline julia> & Parabolico_2 $=b 7[1] .+b 7[2] . *$ dados.d \\
\hline & b7[3].*((dados.d).^2) + b7[4].*dados.i; \\
\hline julia> & $x 8=[$ matrix_um (log.(dados.d)) dados.i $] ;$ \\
\hline julia> & b8 $=\operatorname{inv}\left(x 8^{\prime} \times 8\right)^{*} \times 8^{\prime *}(\log .($ dados.h $)) ;$ \\
\hline julia> & $\begin{array}{l}\text { Potencial_i }=(b 8[1] .+ \text { b8[2].*(log.(dados.d)) .+ } \\
\text { b8[3].*dados.i); }\end{array}$ \\
\hline julia> & $\begin{array}{l}\text { sse_Potencial_i } \quad=\quad \text { sum((Potencial_i- } \\
\left(\log .(\text { dados.h)) }) \cdot{ }^{\wedge} 2\right) ;\end{array}$ \\
\hline julia> & $\begin{array}{l}\text { Syx_Potencial_i } \\
\text { sqrt((sse_Potencial_i./(length((dados.h))-2))); }\end{array}$ \\
\hline julia> & $\begin{array}{l}\text { FCM_Potencial_i } \\
\text { exp.((Syx_Potencial_i.^2).*0.5); }\end{array}$ \\
\hline julia> & $\begin{array}{l}\text { Potencial_1 }=\exp .(\text { b8 }[1] .+ \text { b8[2].* }(\text { log. }(\text { dados.d })) \\
.+ \text { b8[3].*dados.i).*FCM_Potencial_i; }\end{array}$ \\
\hline $\begin{array}{l}\text { julia> } \\
\text { julia> }\end{array}$ & $\begin{array}{l}x 9=\left[\text { matrix_um }\left(1 . /\left((\text { dados.d }) .^{\wedge} 2\right)\right) \text { dados.i }\right] \\
\text { b9 }=\operatorname{inv}\left(x 9^{\prime} \times 9\right)^{*} \times 9^{\prime *}(\text { dados.h }) ;\end{array}$ \\
\hline julia> & 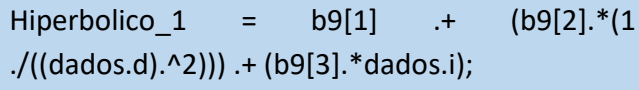 \\
\hline julia> & $x 10=[$ matrix_um (1./(dados.d)) dados.i]; \\
\hline julia> & b10 $=\operatorname{inv}\left(\times 10^{\prime} \times 10\right)^{*} \times 10^{\prime *}(\log .($ dados.h $)) ;$ \\
\hline julia> & $\begin{array}{l}\text { Exponencial_ } \mathrm{i}=\left(\mathrm{b} 10[1] .+\mathrm{b} 10[2] .{ }^{*}(1 . /(\text { dados. } \mathrm{d}))\right. \\
\left..+\mathrm{b} 10[3]^{*} \text { dados. } \mathrm{i}\right) ;\end{array}$ \\
\hline julia> & $\begin{array}{l}\text { sse_exponencial_i } \quad=\quad \operatorname{sum}((\text { Exponencial_i- } \\
(\text { log.(dados.h))).^2); }\end{array}$ \\
\hline julia> & $\begin{array}{l}\text { Syx_exponencial_i } \\
\text { sqrt((sse_exponencial_i./(length((dados.h))-2))); }\end{array}$ \\
\hline julia> & $\begin{array}{l}\text { FCM_exponencial_i } \\
\text { exp((Syx_exponencial_i.^2).*0.5); }\end{array}$ \\
\hline julia> & $\begin{array}{l}\text { Exponencial_1 = exp.(b10[1] .+ b10[2].*(1 ./ } \\
\text { (dados.d)) }\end{array}$ \\
\hline & b10[3]*dados.i).*FCM_exponencial_i; \\
\hline julia> & x11 = [matrix_um (log.(dados.d)) dados.i $]$; \\
\hline julia> & b11 $=\operatorname{inv}\left(x 11^{\prime} \times 11\right)^{*} \times 11^{\prime *}($ dados.h $)$ \\
\hline julia> & Semilogarítmico_i \\
\hline & b11[2].*(log.(dados.d)) .+ b11[3].*dados.i; \\
\hline julia> & x12 = [matrix_um (log.(dados.d)) dados.i]; \\
\hline julia> & b12 = inv $\left(x 12^{\prime} \times 12\right) * x 12^{\prime *}$ log.(dados.h); \\
\hline julia> & $\begin{array}{l}\text { Schumacher_i }=(b 12[1] .+b 12[2] . *(\log .(\text { dados.d })) \\
\left..+b 12[3] .{ }^{*} \text { dados.i }\right) ;\end{array}$ \\
\hline julia> & $\begin{array}{l}\text { sse_Schumacher_i }=\quad \text { sum((Schumacher_i- } \\
\left(\log .(\text { dados.h)) }) \cdot{ }^{\wedge} 2\right) ;\end{array}$ \\
\hline julia> & $\begin{array}{l}\text { Syx_Schumacher_i } \\
\text { sqrt(sse_Schumacher_i./(length((dados.h))-2)); }\end{array}$ \\
\hline julia> & $\begin{array}{l}\text { FCM_Schumacher_i } \\
\text { exp.((Syx_Schumacher_i.^2).*0.5); }\end{array}$ \\
\hline julia> & $\begin{array}{l}\text { Schumacher_1986_2 } \\
\text { b12[2].*(log.(dados.d)) }\end{array}$ \\
\hline & b12[3].*dados.i).*FCM_Schumacher_i; \\
\hline julia> & x13 = [matrix_um (log.(dados.d)) (log.(dados.i))]; \\
\hline julia> & b13 $=\operatorname{inv}\left(x 13^{\prime} \times 13\right)^{*} \times 13^{\prime *}(\log .($ dados.h $)) ;$ \\
\hline julia> & Schumacherhall_i \\
\hline julia> & $\begin{array}{l}b 13[2] . *(\log .(\text { dados.d }))++b 13[3] . *(\log .(\text { dados.i }))) ; \\
\text { sse_Schumacherhall_i }=\operatorname{sum}((\text { Schumacherhall_i- }\end{array}$ \\
\hline
\end{tabular}

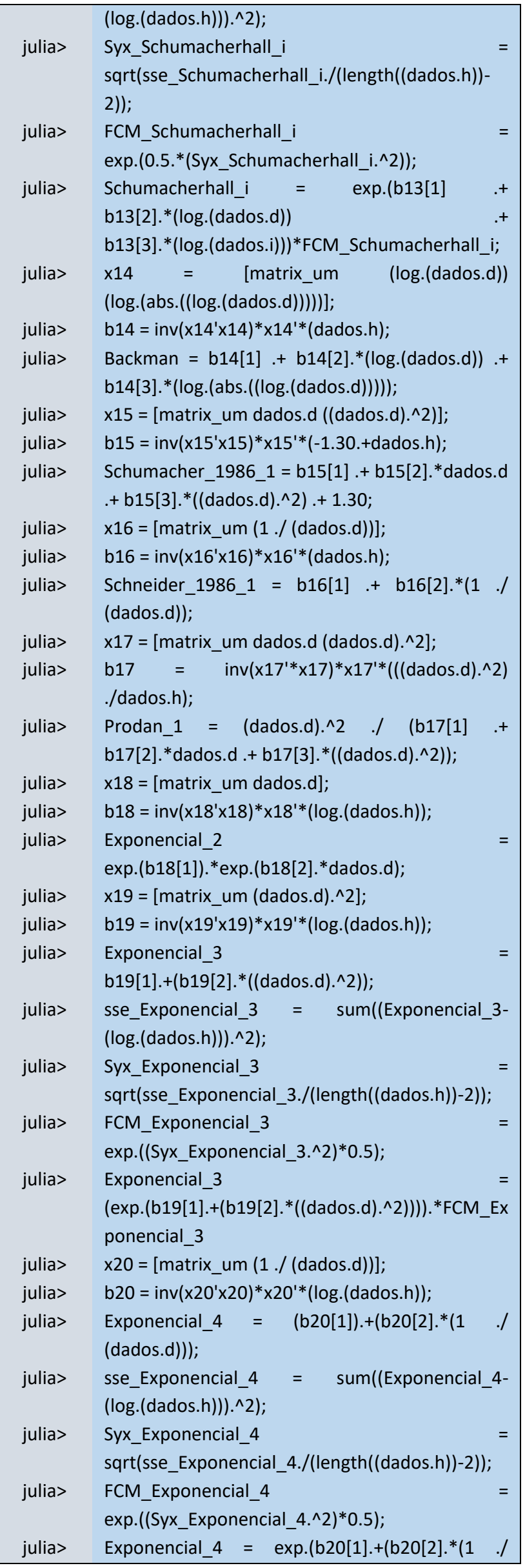




\begin{tabular}{|c|c|}
\hline & (dados.d)))).*FCM_Exponencial_4; \\
\hline julia> & x21 = [matrix_um $(\log .(($ dados.d).^^2))]; \\
\hline julia> & b21 $=\operatorname{inv}\left(\times 21^{\prime} \times 21\right)^{*} \times 21^{\prime *}(\log .($ dados.h $))$ \\
\hline julia> & $\begin{array}{l}\text { Potencial_2 } \\
\text { exp.(b21[1]).*(((dados.d).^2).^b21[2]); }\end{array}$ \\
\hline julia> & x22 = [matrix_um (log.(1 ./ (dados.d)))]; \\
\hline julia> & b22 $=\operatorname{inv}\left(\times 22^{\prime} \times 22\right) * \times 22^{\prime *}(\log .($ dados.h $)) ;$ \\
\hline julia> & $\begin{array}{l}\text { Potencial_3 }=\quad \text { exp.(b22[1]).*((1 } \\
\text { (dados.d)).^b22[2]); }\end{array}$ \\
\hline julia> & x23 = [matrix_um (1./(dados.d))]; \\
\hline julia> & $\begin{array}{l}b 23=\operatorname{inv}\left(\times 23^{\prime} \times 23\right)^{*} \times 23^{\prime *}(1 \quad \text {./ } \text { (dados.h .- } \\
\text { 1.3).^(1/3)); }\end{array}$ \\
\hline julia> & $\begin{array}{l}\text { Petterson }=1.30 .+(1 . /(b 23[1] .+b 23[2] . *(1 . / \\
\left.(\text { dados.d })) .^{\wedge} 3\right) ;\end{array}$ \\
\hline julia> & x24 = [matrix_um dados.d]; \\
\hline julia> & b24 $=\operatorname{inv}\left(\times 24^{\prime} \times 24\right)^{*} \times 24^{\prime} *(\log .($ dados.h $)) ;$ \\
\hline julia> & Berkhout $=$ b24[1].+ dados.d. ${ }^{*}$ b24[2]; \\
\hline julia> & $\begin{array}{l}\text { sse_Berkhout } \quad=\quad \text { sum((Berkhout- } \\
(\text { log.(dados.h))).^2); }\end{array}$ \\
\hline julia> & $\begin{array}{l}\text { Syx_Berkhout } \\
\text { sqrt(sse_Berkhout./(length((dados.h))-2)); }\end{array}$ \\
\hline julia> & FCM_Berkhout $=$ exp.((Syx_Berkhout.^2 $\left.)^{*} 0.5\right)$ \\
\hline julia> & $\begin{array}{l}\text { Berkhout }=\quad \text { exp.(b24[1].+ } \\
\text { dados.d. }{ }^{*} \text { b24[2]).*FCM_Berkhout; }\end{array}$ \\
\hline $\begin{array}{l}\text { Julıa> } \\
\text { julia> }\end{array}$ & $\begin{array}{l}\text { X2b = [matrix_um dados.d (dados.d).^2]; } \\
\text { b25 }=\quad \operatorname{inv}\left(\times 25^{\prime} \times 25\right)^{*} \times 25^{\prime *}\left(\left((\text { dados.d }) .^{\wedge} 2\right)\right.\end{array}$ \\
\hline &.$/$ dados.h); \\
\hline julia> & Naslung_Prodan_2 $=\quad($ dados.d $) . \wedge 2$ \\
\hline & $\begin{array}{l}\left(b 25[1] .+(b 25[2] . * \text { dados.d })+\left(b 25[3] \cdot *^{*}((\text { dados.d }) . \wedge\right.\right. \\
\text { 2))); }\end{array}$ \\
\hline julia> & x26 = [matrix_um (dados.d).^2]; \\
\hline julia> & $\begin{array}{l}\mathrm{b} 26=\operatorname{inv}\left(\times 26^{\prime} \times 26\right)^{*} \times 26^{\prime *}\left(\left((\text { dados.d }) .^{\wedge} 2\right)\right. \\
(\text { dados.h .+1.3)); }\end{array}$ \\
\hline julia> & $\begin{array}{l}\text { Naslung_1 }=\quad(\text { dados.d }) \cdot{ }^{\wedge 2} \\
((\text { b26[1].+(b26[2].*((dados.d).^2))).+1.3); }\end{array}$ \\
\hline julia> & x27 = [matrix_um dados.d (dados.d).^2]; \\
\hline julia> & $\begin{array}{l}\mathrm{b} 27=\operatorname{inv}\left(\times 27^{\prime} \times 27\right)^{*} \times 27^{\prime *}\left(\left((\text { dados.d }) .^{\wedge} 2\right)\right. \\
(\text { dados.h .+1.3)); }\end{array}$ \\
\hline julia> & Naslung_Prodan_1 \\
\hline & $\begin{array}{l}. /((b 27[1] .+(b 27[2] . * \text { dados.d })+(b 27[3] . *((\text { dados.d } \\
) . \wedge 2))) .+1.3)\end{array}$ \\
\hline julia> & x28 = [matrix_um (1./(dados.d))]; \\
\hline julia> & b28 $=\operatorname{inv}\left(\times 28^{\prime} \times 28\right)^{*} \times 28^{\prime *}($ dados.h $)$ \\
\hline julia> & Assmann = b28[1].+ b28[2].*(1./(dados.d)); \\
\hline julia> & x29 = [matrix_um (dados.d).^2]; \\
\hline julia> & b29 $=\operatorname{inv}\left(\times 29^{\prime} \times 29\right)^{*} \times 29^{\prime *}(\log .($ dados.h $))$ \\
\hline julia> & Azevedo_Inh $=(b 29[1] .+b 29[2] . *(($ dados.d $) . \wedge 2)) ;$ \\
\hline julia> & $\begin{array}{l}\text { sse_Azevedo_Inh }=\quad \text { sum((Azevedo_Inh- } \\
(\text { (log.(dados.h))).^2); }\end{array}$ \\
\hline julia> & $\begin{array}{l}\text { Syx_Azevedo_Inh } \\
\text { sqrt(sse_Azevedo_Inh./(length((dados.h))-2)); }\end{array}$ \\
\hline julia> & $\begin{array}{l}\text { FCM_Azevedo_Inh } \\
\text { exp.((Syx_Azevedo_Inh.^2).*0.5); }\end{array}$ \\
\hline julia> & $\begin{array}{l}\text { Azevedo_1999_2 }=\quad \text { exp.(b29[1] } \\
\text { b29[2].*((dados.d).^2)).*FCM_Azevedo_Inh; }\end{array}$ \\
\hline
\end{tabular}

\begin{tabular}{|c|c|}
\hline julia> & $30=[$ matrix_um (sqrt.(dados.d))]; \\
\hline julia> & b30 = inv $(\times 30 ' \times 30) * x 30^{\prime *}($ sqrt.(dados.h)); \\
\hline lia> & Azevedo_1911_5 \\
\hline & b30[2].*(sqrt.(dados.d))).^2; \\
\hline julia> & x31 = [matrix_um (log.(dados.d))]; \\
\hline julia> & b31 $=\operatorname{inv}\left(\times 31^{\prime} \times 31\right)^{*} \times 31^{\prime *}($ sqrt. $(($ dados.h $))) ;$ \\
\hline julia> & Azevedo_1911_6 \\
\hline & b31[2].*(log.(dados.d))).^2; \\
\hline julia> & x32 = [matrix_um (1./(dados.d)) (dado \\
\hline julia> & b32 = inv $\left(\times 32^{\prime} \times 32\right) * \times 32^{\prime *}($ dados.h $)$ \\
\hline julia> & $\begin{array}{l}\text { Azevedo_1911_3 }=\text { b32[1] } .+b 32[2] . *(1 \quad . / \\
(\text { dados.d) })+b 32[3] . *((\text { dados.d } . \wedge 2)\end{array}$ \\
\hline julia> & x33 = [matrix_um dados.d]; \\
\hline julia> & $\begin{array}{l}\text { b33 }=\operatorname{inv}\left(\times 33^{\prime} \times 33\right)^{*} \times 33^{\prime *} \text { (dados.d } \\
\text { (sqrt.(dados.h))); }\end{array}$ \\
\hline julia> & $\begin{array}{l}\text { Azevedo_1999_4 }=\text { (dados.d ./ }(\text { b33[1] .+ } \\
\text { b33[2].*dados.d)).^2; }\end{array}$ \\
\hline julia> & x34 = [matrix_um (1./((dados.d).^^2))]; \\
\hline julia> & b34 = inv $\left(\times 34^{\prime} \times 34\right)^{*} \times 34^{\prime *}($ dados.h $)$ \\
\hline julia> & $4[1] .+b 34[2] * * 1 . /(($ dados \\
\hline julia> & x35 $=[$ mat \\
\hline julia> & b35 = inv $\left(\times 35^{\prime} \times 35\right)^{*} \times 35^{\prime *}(1 . /$ sqrt.(dados.h)); \\
\hline julia> & $\begin{array}{l}\text { renciado_1 = }(1 . /(b 35[1] .+b 35[2] . *(1 . / \\
))) . \wedge 2\end{array}$ \\
\hline julia> & natrix_um (1 ./ (dados.d)) (dados.d).^2]; \\
\hline julia> & b36 $=\operatorname{inv}\left(\times 36^{\prime} \times 36\right)^{*} \times 36^{\prime *}(\log .($ dados.h $)) ;$ \\
\hline julia> & $\begin{array}{l}\text { 1] .+ b36[2].*(1 ./ } \\
\text { d).^2)); }\end{array}$ \\
\hline julia> & \\
\hline julia> & $\begin{array}{l}\text { 11_Inh-(log.(dados.h))).^2); } \\
\text { 1_Inh } \\
\text { 1911_Inh./(length((dados.h))- }\end{array}$ \\
\hline julia> & $\begin{array}{l}\text { FCM_Azevedo_1911_Inh } \\
\text { exp.((Syx_Azevedo_1911_Inh.^2).*0.5); }\end{array}$ \\
\hline julia> & $\begin{array}{l}\text { Azevedo_1911_4 = exp.(b36[1] }+ \text { + b36[2].*(1 ./ } \\
\begin{array}{l}\text { (dados.d) }) \\
.+\end{array}\end{array}$ \\
\hline &.$/(($ dados.d).^2))).*FCM_Azevedo_1911_Inh; \\
\hline julia> & os.d]; \\
\hline lia> & 7)*x37'*(-1.30.+dados.h); \\
\hline julia> & $\begin{array}{l}\text { Schneider_1986_2 }=\text { b37[1] .+ b37[2].*dados.d } \\
.+1.30\end{array}$ \\
\hline julia> & x38 = [matrix_um (1./(dados.d)) dados.d]; \\
\hline julia> & $\left.8^{\prime} \times 38\right)^{*} \times 38^{\prime *}(\mathrm{~d}$ \\
\hline julia> & $\begin{array}{l}\text { Sanquetta_1913 = b38[1].+b38[2].*(1 } \\
(\text { dados.d)).+b38[3].*dados.d; }\end{array}$ \\
\hline julia> & x39 = [matrix_um (dados. \\
\hline julia> & $\begin{array}{l}\text { b39 }=\operatorname{inv}\left(\times 399^{\prime} \times 39\right)^{*} \times 39^{\prime *} \text { (dados.d } \\
(\text { sqrt.(dados.h))); }\end{array}$ \\
\hline julia> & Azevedo_1911_1 \\
\hline $\begin{array}{l}\text { julia } \\
\text { julia }\end{array}$ & $\begin{array}{l}. /(b 39[1] .+b 39[2] . *((\text { dados.d }) . \wedge 2))) .^{\wedge} 2 ; \\
\text { x40 = [matrix_um dados.d]; } \\
\text { b40 } \\
\operatorname{inv}(x 40 ' \times 40)^{*} \times 40^{\prime *} \text { (dados.d./sqrt. }((\text { dados.h) }\end{array}$ \\
\hline julia> & Azevedo_1999_3 \\
\hline
\end{tabular}




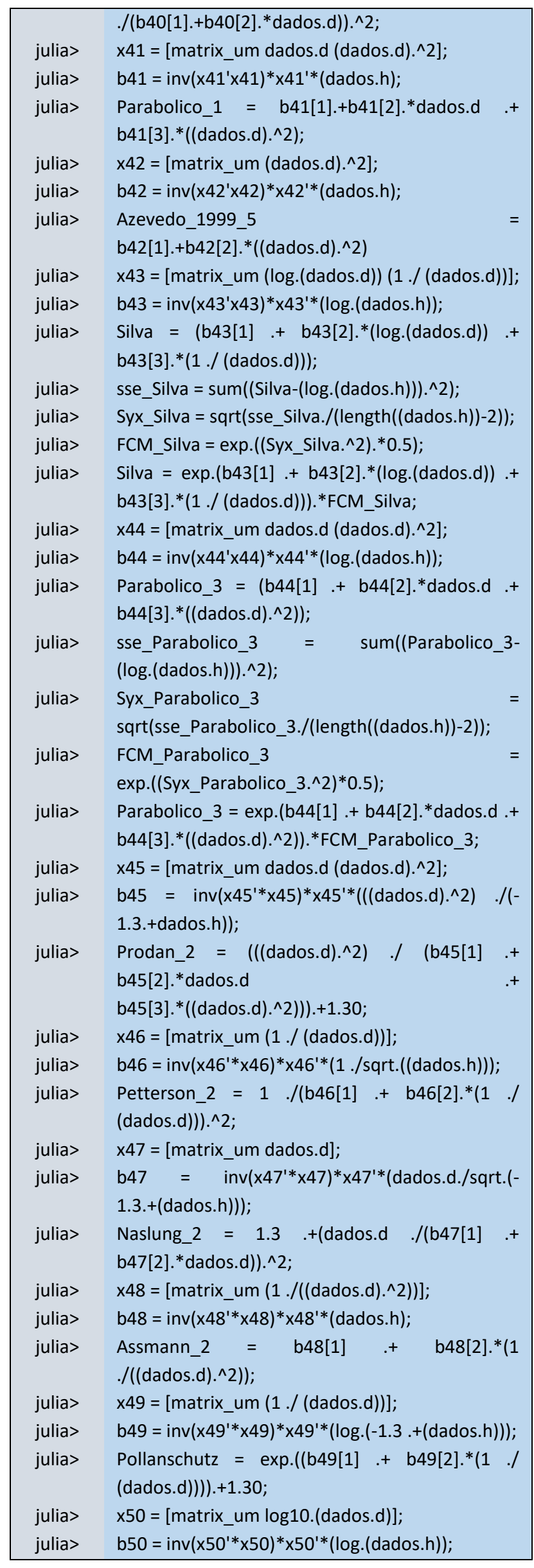

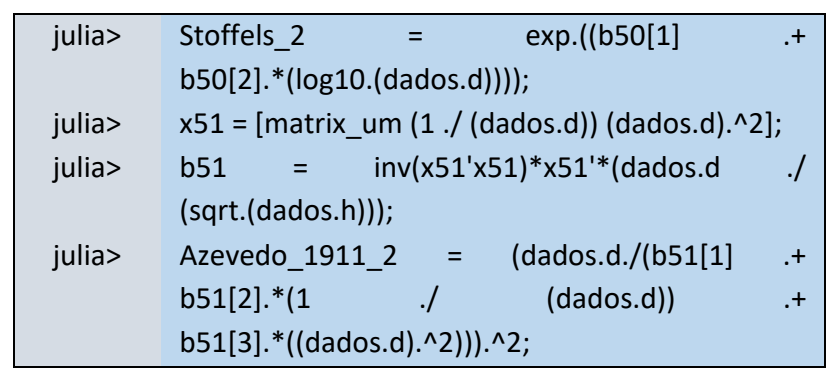

Continuando, o Algoritmo 3 demonstra os processos para elaboração da tabela dos coeficientes $(\beta)$.

Algoritmo 3. Elaboração da tabela com os coeficientes ( $\beta$ ). julia> Nomes =["Linear Simples";"Azevedo et al. (1899) (1)";"Henricksen (1851)";"Curtis (1867)";"Trorey (1831)";"Stoffels \& Soest (1853)";"Parabólico (2)";"Potencial (1)";"Hiperbólico (1)";"Exponencial (1)";"Semilogarítmico (i)";"Schumacher (1986) (2)";"Schumacher-hall (i)";"Backman modificada";"Schumacher (1886) (1)";"Schneider (1886) (1)";"Prodan (1865) (1)";"Exponencial (2)";"Exponencial (3)";"Exponencial (4)";"Potencial (2)";"Potencial (3)";"Petterson";"Berkhout";"Naslung (Prodan) (2)";"Naslung (1)";"Naslung (Prodan) (1)";"Assmann (1)";"Azevedo et al. (1899) (2)";"Azevedo et al. (1911) (5)";"Azevedo et al. (1911) (6)";"Azevedo et al. (1911) (3)";"Azevedo et al. (1899) (4)";"Finger (1892)";"Não Referenciado (1)";"Azevedo et al. (1911) (4)"; "Schneider (1886) (2)";"Sanquetta et al. (1913)";"Azevedo et al. (1911) (1)";"Azevedo et al. (1899) (3)";"Parabólico (1)";"Azevedo et al. (1899) (5)";"Silva (1880)";"Parabolico (3)";"Prodan (1865) (2)";"Petterson (2)";"Naslung (2)";"Assmann (2)";"Pollanschutz";"Stoffels \& Soest (1851) (2)";"Azevedo et al. (1911) (2)"];

julia> beta0

$=[\mathrm{b} 1[1] ; \mathrm{b} 2[1] ; \mathrm{b} 3[1] ; \mathrm{b} 4[1] ; \mathrm{b} 5[1] ; \mathrm{b} 6[1] ; \mathrm{b} 7[1] ; \mathrm{b} 8[1]$ ;b9[1];b10[1];b11[1];b12[1];b13[1];b14[1];b15[1] ;b16[1];b17[1];b18[1];b19[1];b20[1];b21[1];b22[ 1];b23[1];b24[1];b25[1];b26[1];b27[1];b28[1];b2 $9[1] ; b 30[1] ; b 31[1] ; b 32[1] ; b 33[1] ; b 34[1] ; b 35[1] ; b$ 36[1];b37[1];b38[1];b39[1];b40[1];b41[1];b42[1]; b43[1];b44[1];b45[1];b46[1];b47[1];b48[1];b49[1 ];b50[1];b51[1]];

julia> beta1

$=[\mathrm{b} 1[2] ; \mathrm{b} 2[2] ; \mathrm{b} 3[2] ; \mathrm{b} 4[2] ; \mathrm{b} 5[2] ; \mathrm{b} 6[2] ; \mathrm{b} 7[2] ; \mathrm{b} 8[2]$ ;b9[2];b10[2];b11[2];b12[2];b13[2];b14[2];b15[2] ;b16[2];b17[2];b18[2];b19[2];b20[2];b21[2];b22[ 2];b23[2];b24[2];b25[2];b26[2];b27[2];b28[2];b2 9[2];b30[2];b31[2];b32[2];b33[2];b34[2];b35[2];b 36[2];b37[2];b38[2];b39[2];b40[2];b41[2];b42[2]; b43[2];b44[2];b45[2];b46[2];b47[2];b48[2];b49[2 ];b50[2];b51[2]]; 


\begin{tabular}{|c|c|}
\hline julia> & beta2 $\quad$ =["-";"-";"-";"-";b5[3];"- \\
\hline & $\begin{array}{l}\text { ";b7[3];b8[3];b9[3];b10[3];b11[3];b12[3];b13[3]; } \\
\text { b14[3];b15[3];"-";b17[3];"-";"-";"-";"-";"-";"-";"- } \\
\text { ";b25[3];"-";b27[3];"-";"-";"-";"-";b32[3];"-";"-";"- } \\
\text { ";b36[3];"-";"-";"-";"-";b41[3];"- } \\
\text { ";b43[3];b44[3];b45[3];"-";"-";"-";"-";"-";b51[3]]; }\end{array}$ \\
\hline julia> & 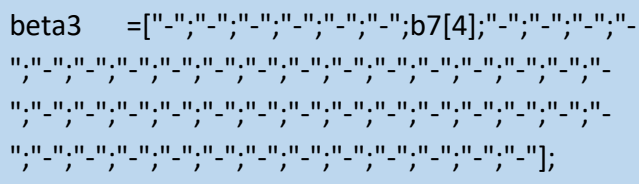 \\
\hline julia> & $\begin{array}{l}\text { nBetas } \\
\text { [length(b1);length(b2);length(b3);length(b4);leng } \\
\text { th(b5);length(b6);length(b7);length(b8);length(b } \\
\text { 9);length(b10);length(b11);length(b12);length(b1 } \\
\text { 3);length(b14);length(b15);length(b16);length(b1 } \\
\text { 7);length(b18);length(b19);length(b20);length(b2 } \\
\text { 1);length(b22);length(b23);length(b24);length(b2 } \\
\text { 5);length(b26);length(b27);length(b28);length(b2 } \\
\text { 9);length(b30);length(b31);length(b32);length(b3 } \\
\text { 3);length(b34);length(b35);length(b36);length(b3 } \\
\text { 7);length(b38);length(b39);length(b40);length(b4 } \\
\text { 1);length(b42);length(b43);length(b44);length(b4 } \\
\text { 5);length(b46);length(b47);length(b48);length(b4 } \\
\text { 9);length(b50);length(b51)]; }\end{array}$ \\
\hline julia> & $\begin{array}{l}\mathrm{B}=\text { DataFrame}(\text { Modelos }=\text { Nomes, Número = } \\
\text { nBetas, } \beta 0=\text { beta0, } \beta 1=\text { beta1, } \beta 2=\text { beta } 2, \beta 3= \\
\text { beta3); } \\
\text { Betas = sort(B); }\end{array}$ \\
\hline
\end{tabular}

O Algoritmo 4 demonstra os processos para elaboração das estatísticas.

Algoritmo 4. Estatísticas e tabela para representar os resultados.

\begin{tabular}{|c|c|}
\hline julia> & $\begin{array}{l}\text { Est }=\text { DataFrame(Altura_Observada= dados.h, } \\
\text { Linear_Simples=Linear, } \\
\text { Azevedo_et_al_1999_1=Azevedo_1999_1,Henric } \\
\text { ksen_1948=Henricksen, Curtis_1967=Curtis, } \\
\text { Trorey_1931=Trorey, } \\
\text { Stoffels_e_Soest_1951_1=Stoffels, } \\
\text { Parabolico_2=Parabolico_2, } \\
\text { Potencial_1=Potencial_1, } \\
\text { Hiperbolico_1=Hiperbolico_1, } \\
\text { Exponencial_1=Exponencial_1, } \\
\text { Semilogaritmico_i=Semilogarítmico_i,Schumache } \\
\text { r_1986_2=Schumacher_1986_2,Schumacherhall } \\
\text { i=Schumacherhall_i, } \\
\text { Backman_modificada=Backman, } \\
\text { Schumacher_1986_1=Schumacher_1986_1, } \\
\text { Schneider_1986_1=Schneider_1986_1, } \\
\text { Prodan_1965_1=Prodan_1, } \\
\text { Exponecencial_2=Exponencial_2, } \\
\text { Exponencial_3=Exponencial_3, } \\
\text { Exponencia_4=Exponencial_4, } \\
\text { Potencial_2=Potencial_2, }\end{array}$ \\
\hline
\end{tabular}

Potencial_3=Potencial_3, Petterson=Petterson, Berkhout=Berkhout, Naslung_Prodan_2= Naslung_Prodan_2, Naslung=Naslung_1, Naslung_Prodan_1=Naslung_Prodan_1,

Assmann_1=Assmann,

Azevedo_et_al_1999_2=Azevedo_1999_2, Azevedo_et_al_1911_5=Azevedo_1911_5, Azevedo_et_al_1911_6=Azevedo_1911_6, Azevedo_et_al_1911_3=Azevedo_1911_3, Azevedo_et_al_1999_4=Azevedo_1999_4, Finger=Finger,

Nao_Referenciado_1=nao_referenciado_1, Azevedo_et_al_1911_4

Azevedo_1911_4,Schneider_1986_2 Schneider_1986_2,Sanquetta_et_al_1913=Sanqu etta_1913,

Azevedo_et_al_1911_1=Azevedo_1911_1,Azeve do_et_al_1999_3=Azevedo_1999_3,Parabolico_ 1=Parabolico_1,Azevedo_et_al_1999_5=Azevedo _1999_5,Silva_1980=Silva,Parabolico_3=Paraboli co_3,Prodan_1965_2=Prodan_2,Petterson_2=Pe tterson_2,Naslung_2=Naslung_2,Assmann_2=Ass mann_2,Pollanschutz=Pollanschutz,Stoffels_e_S oest_1951_2=Stoffels_2,Azevedo_et_al_1911_2 =Azevedo_1911_2);

julia $>$ Estimativas $=\operatorname{sort}($ Est $)$

julia> $\quad \mathrm{Y}=$ dados.h

julia> Yhat $=$ Est[:,2:end $]$

julia> $\quad p=s i z e(Y h a t, 2)$

julia> ei $=[Y-Y h a t[:, i]$ for $i$ in $1: p]$

julia> ebar $=[(\operatorname{sum}(Y-Y h a t[:, i]) /$ length $(Y))$ for $\mathrm{i}$ in 1:p $]$

julia $>$ ebarp $\quad=\quad[(($ sum $(Y-$ Yhat $[:, i]) /$ length $(Y)) /$ mean $(Y)) * 100)$ for $i$ in 1:p]

julia $>\quad$ Se $\quad=\quad[\operatorname{sqrt}((\operatorname{sum}(((Y-Y h a t[:, i])--((\operatorname{sum}(Y-$ Yhat $[:, i]) /$ length $\left.\left.(Y)))) .^{\wedge} 2\right)\right) /($ length $\left.(Y)-1)\right)$ for $i$ in 1:p]

julia $\quad$ Sep $=[((\operatorname{sqrt}((\operatorname{sum})((Y-Y h a t[:, i])--((\operatorname{sum}(Y-$ Yhat[:,i])/length(Y)))).^2))/(length(Y)-

$1)) /$ mean $(Y)) * 100)$ for $i$ in $1: p]$

julia $\quad m x=[\operatorname{sqrt}(((\operatorname{sqrt}((\operatorname{sum})((Y-Y h a t[:, i]) \cdot-((\operatorname{sum}(Y-$ Yhat[:,i])/length(Y)))).^2))/(length(Y)-1)))^2)+ $\left.\left(((\operatorname{sum}(Y-Y h a t[:, i]) / \text { length }(Y)))^{\wedge} 2\right)\right)$ for $i$ in 1:p]

julia $\quad m x p=[(((\operatorname{sqrt}(((\operatorname{sqrt})(\operatorname{sum}(((Y-Y h a t[:, i]))-((\operatorname{sum}(Y-$ Yhat $[:, i]) /$ length(Y)))).^2))/(length(Y)-1)))^2)+ ( ( $($ sum $(Y-$

Yhat $[:, i]) /$ length $\left.\left.\left.(Y)))^{\wedge} 2\right)\right)\right) /($ mean $\left.\left.(Y))\right)^{*} 100\right)$ for $\mathrm{i}$ in 1:p]

julia> ei $=[Y-Y h a t[:, i]$ for $\mathrm{i}$ in 1:p]

julia $>\quad Z=$ DataFrame(Modelos=Nomes, Viés=ebar, Viés $P=e b a r p, \quad P r e c i s a ̃ o=S e, \quad P r e c i s a ̃ o P=S e p$, Acurácia $=m x$, Acurácia $P=m x p)$

julia $\quad$ Estatisticas $=\operatorname{sort!(Z,~[:AcuráciaP])~\# rev=true~se~}$ for do maior para o menor

O Algoritmo 5 foi desenvolvido para exportar os 
resultados para arquivo .xls (EXCEL).

Algoritmo 5. Exportar os resultados para EXCEL.

\begin{tabular}{|c|c|}
\hline julia> & Exportar estatísticas para o Excel \\
\hline \multirow[t]{5}{*}{ julia> } & $\begin{array}{l}\text { XLSX.openxIsx("C:/Pasta1/Pasta2/PastaN/Estatist } \\
\text { icas.xIsx", mode="w") do xf } \\
\text { sheet = xf[1] } \\
\text { XLSX.rename!(sheet, "VPA") } \\
\text { sheet["A1"] = ["Modelos"] } \\
\text { sheet["B1"] = ["VIES"] } \\
\text { sheet["C1"] = ["VIESP"] } \\
\text { sheet["D1"] = ["PRECISÃO"] } \\
\text { sheet["E1"] = ["PRECISÃOP"] } \\
\text { sheet["F1"] = ["ACURACIA"] } \\
\text { sheet["G1"] = ["ACURACIAP"] }\end{array}$ \\
\hline & sheet["A2:G54"] = [Estatisticas.Modelos \\
\hline & Estatisticas.Viés \\
\hline & Estatisticas.Precisão \\
\hline & $\begin{array}{l}\text { Estatisticas.Acurácia Estatisticas.AcuráciaP] } \\
\text { end }\end{array}$ \\
\hline julia> & \#Exportar tabela de parâmetros para o Excel \\
\hline julia> & XLSX.openxIsx("C:/ \\
\hline & $\begin{array}{l}\text { Pasta1/Pasta2/PastaN/Betas.xlsx", mode="w") } \\
\text { do } x f \\
\text { sheet }=x f[1]\end{array}$ \\
\hline & XLSX.rename!(sheet, "VPA") \\
\hline & sheet["A1"] = ["Modelos"] \\
\hline & sheet["B1"] = ["Número"] \\
\hline & sheet["C1"] = [" $\beta 0 "]$ \\
\hline & sheet["D1"] = ["ß1"] \\
\hline & sheet["E1"] = ["ß2"] \\
\hline & sheet["F1"] = ["ß3"] \\
\hline & sheet["A2:F54"] = [Betas.Modelos Betas.Número \\
\hline & $\begin{array}{l}\text { Betas. } \beta 0 \text { Betas. } \beta 1 \text { Betas. } \beta 2 \text { Betas. } \beta 3] \\
\text { end }\end{array}$ \\
\hline
\end{tabular}

O Algoritmo 6 apresenta a elaboração dos gráficos de resíduo e dispersão apresentadas para auxiliar nos resultados.

Algoritmo 6. Desenvolvimento dos gráficos de resíduo e dispersão.

\begin{tabular}{ll|} 
julia> & \#Gráfico de Resíduo \\
julia> & Resíduo = dados.h - Nome_do_Modelo; \\
julia> & plot(dados.d, residuo, "b.") \\
julia> & xlabel("Diâmetro à altura do peito (cm)") \\
julia> & ylabel("Resíduo (m)") \\
julia> & title("TITULO") \\
julia> & ylim(-20, 20) \\
julia> & xlim(0,25) \\
julia> & plt.plot([0, 25],[0,0],'k') \\
julia> & \\
julia> & \#Gráfico de Dispersão \\
julia> & scatter(dados.d, dados.h, facecolor= "none", \\
& edgecolors="orange", s=20, label="Observado")
\end{tabular}

$\begin{array}{ll}\text { julia> } & \text { scatter(dados.d, Nome_do_Modelo, facecolor= } \\ & \text { "none", edgecolors="blue", s=20, label= } \\ & \text { "Estimado") } \\ \text { julia> } & \text { xlabel("Diâmetro à altura do peito (cm)") } \\ \text { julia> } & \text { ylabel("Altura (m)") } \\ \text { julia> } & \text { legend(prop=Dict("size"=>10)) } \\ \text { julia> } & \text { title("TITULO") }\end{array}$

\section{RESULTADOS E DISCUSSÃO}

As estatísticas descritivas obtidas para os dados utilizados no ajuste dos modelos estão descritas na tabela 3. Ao se avaliar a variável altura observou-se um coeficiente de variação (CV\%) de 46,18, 16,65, 19,60 e 18,06 para as classes: Jovem, Média Inicial, Média Avançada e Madura, respectivamente. Pode-se observar a redução nos valores das demais idades para a classe Jovem.

De modo geral essa maior variação na classe de idade Jovem se dá pela alta variabilidade dos exemplares, e pela variável que está sendo mensurada, que apresenta uma alta variância no início do crescimento das árvores.

Tabela 3. Estatísticas descritivas das variáveis diâmetro à altura do peito $(\mathrm{cm})$ e altura total $(\mathrm{m})$ por grupos de equações e classes de idade dos povoamentos de acácia-negra provenientes do Rio Grande do Sul, Brasil.

\begin{tabular}{|c|c|c|}
\hline \multirow{2}{*}{ Grupo } & \multicolumn{2}{|c|}{ Jovem } \\
\hline & $\mathrm{d}(\mathrm{cm})$ & $h(m)$ \\
\hline $\bar{x}$ & 5,09 & 5,97 \\
\hline CV\% & 54,18 & 43,11 \\
\hline IC superior & 5,45 & 6,31 \\
\hline IC inferior & 4,72 & 5,63 \\
\hline \multirow{2}{*}{ Grupo } & \multicolumn{2}{|c|}{ Média Inicial } \\
\hline & $\mathrm{d}(\mathrm{cm})$ & $h(m)$ \\
\hline$\overline{\bar{x}}$ & 9,27 & 11,91 \\
\hline CV\% & 24,49 & 15,19 \\
\hline IC superior & 9,60 & 12,17 \\
\hline IC inferior & 8,95 & 11,65 \\
\hline \multirow{2}{*}{ Grupo } & \multicolumn{2}{|c|}{ Média Avançada } \\
\hline & $\mathrm{d}(\mathrm{cm})$ & $h(m)$ \\
\hline$\overline{\bar{x}}$ & 11,39 & 15,13 \\
\hline CV\% & 32,30 & 20,38 \\
\hline IC superior & 11,94 & 15,59 \\
\hline IC inferior & 10,84 & 14,67 \\
\hline \multirow{2}{*}{ Grupo } & \multicolumn{2}{|c|}{ Madura } \\
\hline & $\mathrm{d}(\mathrm{cm})$ & $h(m)$ \\
\hline $\bar{x}$ & 12,98 & 16,42 \\
\hline CV\% & 31,08 & 18,12 \\
\hline IC superior & 13,54 & 16,84 \\
\hline IC inferior & 12,41 & 16,00 \\
\hline
\end{tabular}

Em que: $\quad \overline{\mathrm{x}}=$ média; $\quad \mathrm{CV} \%=$ coeficiente de variação (\%); IC inferior = intervalo de confiança inferior; $\quad$ IC superior $=$ intervalo de confiança superior. 


\section{Ajuste dos modelos hipsométricos}

Os coeficientes dos modelos hipsométricos, para a estimativa da altura total ( $h$ ) estão apresentados na Tabela 4. De modo a melhorar a apresentação do conteúdo, foram disponibilizados os 5 melhores modelos de cada Classe de Idade. Já os resultados com todos os modelos são apresentados no Apêndice.

Tabela 4. Parâmetros Betas dos modelos ajustados para cálculo da altura total de indivíduos de acácianegra.

\begin{tabular}{|c|c|c|c|c|c|}
\hline \multirow{2}{*}{ Modelos } & \multirow[b]{2}{*}{ GL } & \multicolumn{4}{|c|}{ Jovem } \\
\hline & & $\beta_{0}$ & $\beta_{1}$ & $\boldsymbol{\beta}_{2}$ & $\beta_{3}$ \\
\hline Parabólico (2) & 4 & $-0,8146$ & 0,9971 & $-0,0326$ & 1,8692 \\
\hline \multicolumn{6}{|l|}{ Schumacher-hall } \\
\hline (i) & 3 & 0,7195 & 0,5175 & 0,5780 & - \\
\hline Potencial (1) & 3 & 0,2980 & 0,5209 & 0,4196 & - \\
\hline \multicolumn{6}{|l|}{ Schneider (1986) } \\
\hline (2) & 2 & 0,1085 & 0,8946 & 0,0087 & - \\
\hline Prodan (1965) (2) & 3 & 1,5599 & 0,2542 & 0,0857 & - \\
\hline \multirow{2}{*}{ Modelos } & & \multicolumn{4}{|c|}{ Média Inicial } \\
\hline & GL & $\beta_{0}$ & $\beta_{1}$ & $\boldsymbol{\beta}_{2}$ & $\beta_{3}$ \\
\hline Parabólico (2) & 4 & 6,3283 & 1,5472 & $-0,0523$ & $-1,4550$ \\
\hline Exponencial (1) & 3 & 3,2009 & $-3,3490$ & $-0,1275$ & - \\
\hline Semilogarítmico (i) & 3 & 4,5708 & 4,9194 & $-1,2737$ & - \\
\hline \multicolumn{6}{|l|}{ Schumacher-hall } \\
\hline (i) & 3 & 1,7061 & 0,4660 & $-0,2673$ & - \\
\hline Trorey (1932) & 3 & 2,1947 & 1,4908 & $-0,0454$ & - \\
\hline \multirow{2}{*}{ Modelos } & & \multicolumn{4}{|c|}{ Média Avançada } \\
\hline & GL & $\beta_{0}$ & $\beta_{1}$ & $\boldsymbol{\beta}_{2}$ & $\beta_{3}$ \\
\hline Exponencial (1) & 3 & 2,341 & $-4,1524$ & 0,1471 & - \\
\hline Semilogarítmico (i) & 3 & $-12,5047$ & 6,8762 & 2,1564 & - \\
\hline Parabólico (2) & 4 & $-11,065$ & 1,9049 & $-0,0564$ & 2,376 \\
\hline Potencial (1) & 3 & 0,8138 & 0,5495 & 0,1102 & - \\
\hline $\begin{array}{l}\text { Schumacher-hall } \\
\text { (i) }\end{array}$ & 3 & 0,424 & 0,5498 & 0,5841 & - \\
\hline
\end{tabular}

\begin{tabular}{cccccc}
\hline \multirow{2}{*}{ Modelos } & & \multicolumn{4}{c}{ Madura } \\
\cline { 3 - 6 } & $\mathbf{G L}$ & $\boldsymbol{\beta}_{\mathbf{0}}$ & $\boldsymbol{\beta}_{\mathbf{1}}$ & $\boldsymbol{\beta}_{\mathbf{2}}$ & $\boldsymbol{\beta}_{\mathbf{3}}$ \\
\hline Exponencial (1) & 3 & 3,0048 & $-5,5757$ & 0,0254 & - \\
Parabólico (2) & 4 & $-3,0917$ & 1,7957 & $-0,0437$ & 0,422 \\
Prodan (1965) (2) & 3 & 2,1633 & 0,0489 & 0,0467 & - \\
Prodan (1965) (1) & 3 & 1,5904 & 0,0774 & 0,043 & - \\
Naslung (Prodan) & & 1,5904 & 0,0774 & 0,043 & - \\
(2) & 3 & & & \\
\hline
\end{tabular}

Em que: $\mathrm{d}=$ diâmetro altura do peito $(\mathrm{cm}) ; \quad \mathrm{h}=$ altura total $(\mathrm{m}) ; \mathrm{GL}=\mathrm{Grau}$ de liberdade; $\beta_{0}, \beta_{1}, \beta_{2}$ e $\beta_{3}$, parâmetros;

Os resultados do Viés, Precisão e Acurácia para os 5 melhores modelos de cada Classe de Idade foram apresentados na Tabela 5.

As estatísticas resultantes dos ajustes dos modelos expressas na Tabela 5 demostraram que para as duas classes de idades (Jovem e Média Inicial), o melhor modelo empregado foi o Parabólico 2 (Tabela 2), com os valores de $\bar{e}_{\%} 1,96 \mathrm{E}^{-12}$ e $-1,96 \mathrm{E}^{-12}, \mathrm{~S}_{\mathrm{e}} \mathrm{8,99}$ e 7,76, e $\mathrm{m}_{\mathrm{x} \%} 8,99$ e 7,76, e para as classes restantes o modelo que demonstrou melhores resultados foi o Exponencial 1 (Tabela 2) apresentando, $\bar{e}_{\%}-0,01$ e $-0,01, \mathrm{~S}_{\mathrm{e}} \%$ 7,86 e 7,03, e $m_{x \%} 7,86$ e 7,03, respectivamente.

Da mesma forma, os resultados obtidos na observação da distribuição dos resíduos em relação à altura estimada demonstram que os modelos demonstraram superioridade em seus ajustes, para todas as classes: Jovem, Médio Inicial, Médio Avançada e Madura (Figura 1).

Ao analisar a Tabela 3 e os gráficos da Figuras 1, de modo geral, observou-se que os modelos testados apresentaram ajustes variados com valores de Viés (\%) variando entre $-0,0280$ a 0,52. Contudo, ao avaliar o comportamento das distribuições dos resíduos, juntamente com as estatísticas selecionaram-se os modelos de Parabólico (2) para os classes de idade Jovem e Média Inicial, e os modelos Exponencial (1) para os classes de idade Média Avançada e Madura, pois apresentaram os melhores ajustes. A variação dos valores obtidos foi relativamente baixa, tendo em vista que o $\mathrm{S}_{\mathrm{e}} \%$ que variou entre $7,03 \%$ a $10,10 \%$, e o $m_{x} \%$ apresentou uma variação similar com valores entre 7,03 a 10,15\%.

Caldeira et al. (2002) estudaram povoamentos de acácia-negra com idade entre 2 e 8 anos e as equações hipsométricas apresentaram $\mathrm{Se}$ entre 0,07 a 1,98. Os autores afirmam que a melhor equação foi uma logarítmica com $S_{e}$ de 0,07 , e em sua distribuição de resíduos não apresentou tendências de superestimar ou subestimar as alturas dentro da amplitude de diâmetros observados. Uma das causas da diferença entre os valores residuais da classe de idade Jovem se dá por apresentar dados de parcelas diferentes, sendo assim as características dos grupos se alteraram, valendo-se principalmente do fator idade.

Sanquetta et al. (2014b) apresentaram resultados para indivíduos de acácia-negra aos sete anos de idade provenientes do Rio Grande do Sul, onde, o $\mathrm{S}_{\mathrm{e}} \%$ variou de $0,98 \%$ a $16,21 \%$. O modelo que melhor se ajustou apresentou $\mathrm{S}_{\mathrm{e} \%}$ de 0,98.

Müller et al. (2014) apresentaram equações para Acacia mangium aos 10 anos de idade, com $\mathrm{R}^{2}$ variando entre 0,84 e 0,93 e $m_{x}$ com variação de 2,44 a 3,40. Para os autores as equações de Max \& Burkhart e Parresol geraram os melhores modelos com $m_{x}$ de 2,56 e 2,44, respectivamente. 
Tabela 5. Viés, precisão e acurácia para os modelos para cálculo da altura total de indivíduos de acácia-negra.

\begin{tabular}{|c|c|c|c|c|c|c|}
\hline \multirow{2}{*}{ Modelos } & \multicolumn{6}{|c|}{ Jovem } \\
\hline & $\overline{\boldsymbol{e}}$ & $\overline{\boldsymbol{e}}_{\%}$ & $S_{e}$ & $\boldsymbol{S}_{\boldsymbol{e} \%}$ & $\boldsymbol{m}_{x}$ & $\boldsymbol{m}_{\boldsymbol{x} \%}$ \\
\hline Parabólico (2) & $1,0294 \mathrm{E}^{-13}$ & $1,96 \mathrm{E}^{-12}$ & 0,4710 & 8,99 & 0,4710 & 8,99 \\
\hline Schumacher-hall (i) & $-0,0035$ & $-0,07$ & 0,4907 & 9,37 & 0,4907 & 9,37 \\
\hline Potencial (1) & $-0,0035$ & $-0,07$ & 0,4907 & 9,37 & 0,4907 & 9,37 \\
\hline Schumacher (1986) (2) & $-0,0035$ & $-0,07$ & 0,4907 & 9,37 & 0,4907 & 9,37 \\
\hline Prodan (1965) (2) & 0,0494 & 0,94 & 0,5293 & 10,10 & 0,5316 & 10,15 \\
\hline \multirow{2}{*}{ Modelos } & \multicolumn{6}{|c|}{ Média Inicial } \\
\hline & $\overline{\boldsymbol{e}}$ & $\overline{\boldsymbol{e}}_{\%}$ & $S_{e}$ & $S_{e \%}$ & $m_{x}$ & $\boldsymbol{m}_{\boldsymbol{x} \%}$ \\
\hline Parabólico (2) & $2,3079 \mathrm{E}^{-13}$ & $1,96 \mathrm{E}^{-12}$ & 0,9116 & 7,76 & 0,9116 & 7,76 \\
\hline Exponencial (1) & 0,0009 & 0,01 & 0,9289 & 7,91 & 0,9289 & 7,91 \\
\hline Semilogarítmico (i) & $-3,2045 \mathrm{E}^{-13}$ & $-2,73 \mathrm{E}^{-12}$ & 0,9334 & 7,95 & 0,9334 & 7,95 \\
\hline Schumacher-hall (i) & $-0,0081$ & $-0,07$ & 0,9954 & 8,47 & 0,9954 & 8,47 \\
\hline Trorey (1932) & $4,0454 \mathrm{E}^{-13}$ & $3,44 \mathrm{E}^{-12}$ & 0,9962 & 8,48 & 0,9962 & 8,46 \\
\hline \multirow{2}{*}{ Modelos } & \multicolumn{6}{|c|}{ Média Avançada } \\
\hline & $\overline{\boldsymbol{e}}$ & $\overline{\boldsymbol{e}}_{\%}$ & $S_{e}$ & $S_{e \%}$ & $m_{x}$ & $\boldsymbol{m}_{\boldsymbol{x} \%}$ \\
\hline Exponencial (1) & $-0,0009$ & $-0,01$ & 1,2038 & 7,86 & 1,2038 & 7,86 \\
\hline Semilogarítmico (i) & $9,3231 \mathrm{E}^{-13}$ & $6,0843 \mathrm{E}^{-12}$ & 1,2511 & 8,16 & 1,2511 & 8,16 \\
\hline Parabólico (2) & $9,3681 \mathrm{E}^{-13}$ & $6,1137 \mathrm{E}^{-12}$ & 1,2537 & 8,18 & 1,2537 & 8,18 \\
\hline Potencial (1) & $-0,0280$ & $-0,1830$ & 1,3708 & 8,95 & 1,3710 & 8,95 \\
\hline Schumacher (1986) (2) & $-0,0280$ & $-0,1830$ & 1,3708 & 8,95 & 1,3710 & 8,95 \\
\hline \multirow{2}{*}{ Modelos } & \multicolumn{6}{|c|}{ Madura } \\
\hline & $\overline{\boldsymbol{e}}$ & $\overline{\boldsymbol{e}}_{\%}$ & $S_{e}$ & $\boldsymbol{S}_{\boldsymbol{e} \%}$ & $m_{x}$ & $\boldsymbol{m}_{\boldsymbol{x} \%}$ \\
\hline Exponencial (1) & $-0,0019$ & $-0,01$ & 1,1591 & 7,03 & 1,1591 & 7,03 \\
\hline Parabólico (2) & $9,2465 \mathrm{E}^{-13}$ & $5,61 \mathrm{E}^{-12}$ & 1,1599 & 7,04 & 1,1599 & 7,04 \\
\hline Prodan (1965) (2) & 0,0933 & 0,57 & 1,1626 & 7,05 & 1,1663 & 7,08 \\
\hline Prodan (1965) (1) & 0,0850 & 0,52 & 1,1673 & 7,08 & 1,1704 & 7,10 \\
\hline Naslung (Prodan) (2) & 0,0850 & 0,52 & 1,1673 & 7,08 & 1,1704 & 7,10 \\
\hline
\end{tabular}

Em que: $\bar{e}$ é o víes absoluto, $\bar{e}_{\%}$ é o viés relativo, $S_{e}$ é a precisão absoluta, $S_{e \%}$ é a precisão relativa, $m_{x}$ é a acurácia absoluta e $m_{x \%}$ é a acurácia relativa.

Ao analisar o Viés, Ribeiro et al. (2015) apresenta valores de 0,7276 a 2,3453 para 14 modelos hipsométricos de Eucalyptus sp. Costa et al. (2014) descrevem valores de $\bar{e}-0,0858$ a 0,0093 para povoamentos de Araucaria angustifólia.

Soares (2015) descreveu valores de $\bar{e}-0,020$ a 0,254 , Se 0,224 a 2,259 e $m_{\times \%} 16,73$ a $17,07 \%$ para povoamento de Acácia-negra.

Pode-se observar que não houve similaridade entre os trabalhos, variando modelos e resultados. Essa diferença pode ser atribuída à idade entre os povoamentos, condições inerentes ao sítio, bem como procedência (CALDEIRA et al., 2002). Dessa forma compreende-se que as relações hipsométricas são influenciadas pela idade, sítio, densidade e espécie (LOETSCH et al., 1973; FINGER, 1992; SCOLFORO, 1999; HUSCH e BEERS, 2002).

\section{Validação da melhor equação}

Obteve-se um valor para o Qui-quadrado $\left(\chi^{2}\right)$ calculado para as classes de idade: Jovem $(2,0239)$, Média Inicial
(1,0364), Média Avançada $(118,1416)$ e Madura $(0,6158)$. Não sendo significativos para o valor $\chi^{2}$ tabelado 30,1435 com grau de liberdade (GL) 19 e 95\% para o nível de significância. As Precisão de 12,02\%, 6,78\%, 67,61\% e $4,65 \%$, respectivamente. Nessa questão o único modelo que teve um valor significativo foi para a classe Média Avançada.

A respeito da validação foi possível estabelecer que o modelo de Parabólico (2) e Exponencial (1) apresentaram resultados que permitem o uso dos mesmos para estimar alturas de outras árvores de acácia-negra, exceto para o grupo Média Avançada, os modelos para essa idade não podem ser utilizados em outras bases de dados. Quanto às curvas hipsométricas, observou-se que elas apresentaram comportamentos similares aos apresentados por Sanquetta et al. (2014b) para acácia-negra no estado do Rio Grande do Sul. 

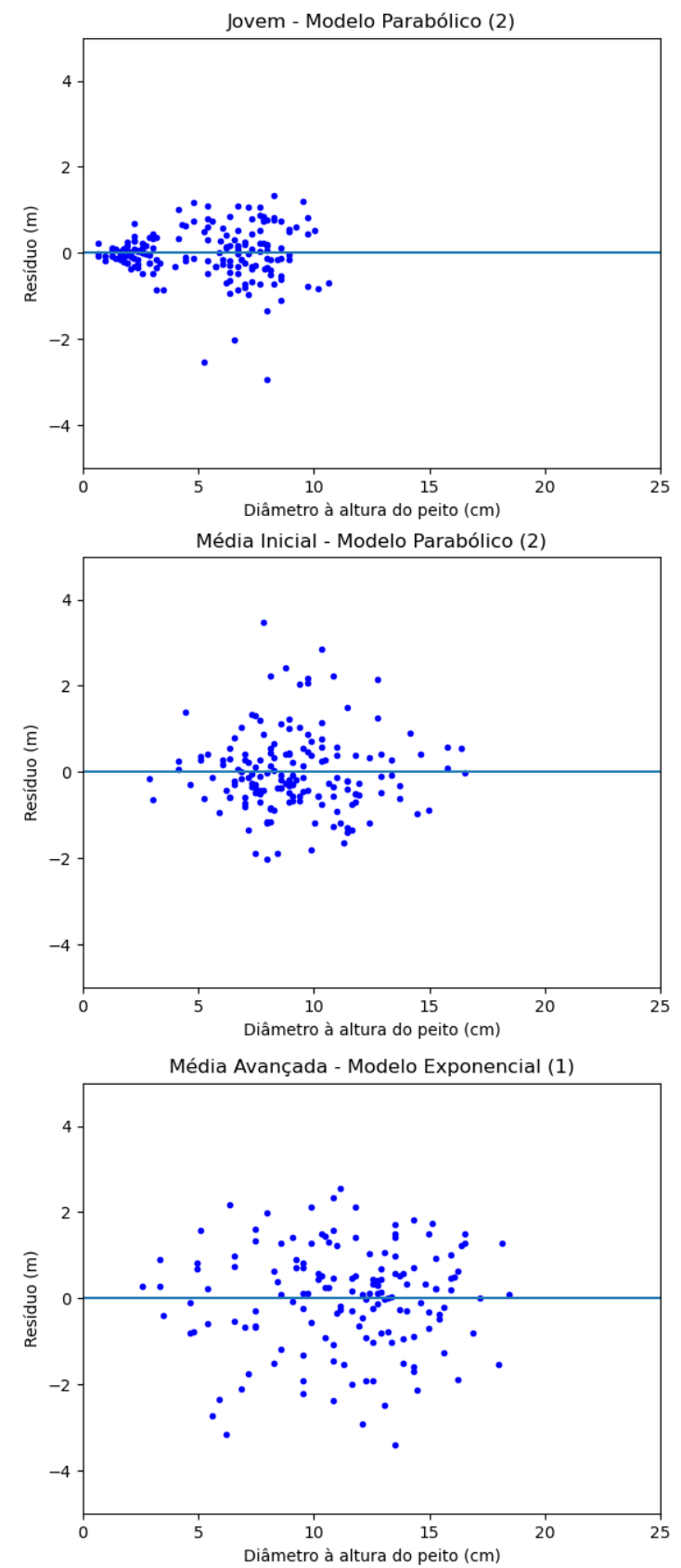

Madura - Modelo Exponencial (1)

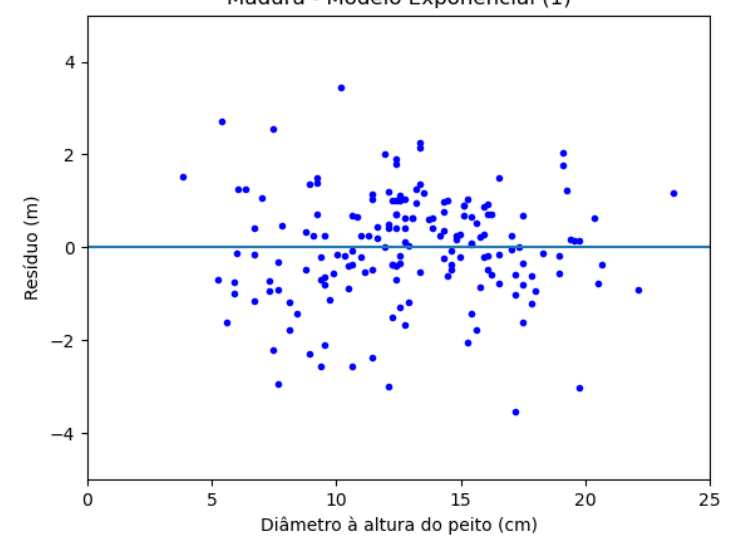

Figura 1. Distribuição dos resíduos da altura (\%) em relação à altura estimada $(\mathrm{m})$ para indivíduos de acácianegra para as classes Jovem, Média Inicial, Média Avançada e Madura.

\section{Curva hipsométrica}

Com o intuito de demonstrar a dispersão dos indivíduos coletados em relação às curvas hipsométricas obtidas pelo modelo Parabólico (2) para as idades Jovem e Média Inicial e Exponencial (1) para Média Avançada e Madura, ao se comparar com os dados observados. Optou-se pelo uso do método gráfico para melhor representar essa relação (Figura 2).

Quanto ao uso da Linguagem de Programação Julia, foi possível gerar toda programação. O maior diferencial dessa Linguagem de Programação decorre do fato de apresentar uma escrita que se assemelha as fórmulas matemáticas, facilitando o entendimento e a reprodução dos códigos base. Outro fator a se levar em conta é a capacidade de expandir a lista de modelos que o programador irá utilizar, o que não ocorre em certos programas que já possuem um número limitado de modelos indexado.

A respeito da Tabela 6, onde observou-se os resultados dos reagrupamentos das bases de dados, e se observou o aumento da seletividade no agrupamento dos dados não apresenta uma melhora nos modelos. Uma explicação para esse fator, se dá pela redução no número de variáveis o que diminui a representatividade da base de dados.

Sendo assim, a Linguagem de Programação Julia foi apta a realizar as demandas exigidas no artigo, sendo uma alternativa a softwares e Linguagens de Programação utilizadas para a mesma finalidade.

\section{CONCLUSÕES}

O modelo que melhor se ajustou para estimar altura total de indivíduos da espécie Acacia mearnsii, foi o Parabólico (2) para as idades Jovem e Média Inicial e o Exponencial (1) para as idades Média Avançada e Madura, apresentando $\bar{e}$ de $1,96 \mathrm{E}^{-12}, 1,96 \mathrm{E}^{-12},-0,01$ e $-0,01$ para as Classes de Idades: Jovem, Média Inicial, Média Avançada e Madura, respectivamente. Para $S_{e \%}$ com valores de 8,99 , $7,76,7,86$ e 7,03 e $m_{x \%} 8,99,7,76,7,86$ e 7,03, respectivamente.

O procedimento de validação determina que os modelos também apresentem aptidão para serem utilizados com indivíduos que não pertenceram ao ajuste das equações, com exceção da classe Média Avançada.

Confirmando a hipótese proposta no início do trabalho a Linguagem de Programação Julia atendeu a todas as demandas propostas pelos autores e é uma alternativa para programadores e Engenheiros Florestais na elaboração de modelos hipsométricos. 


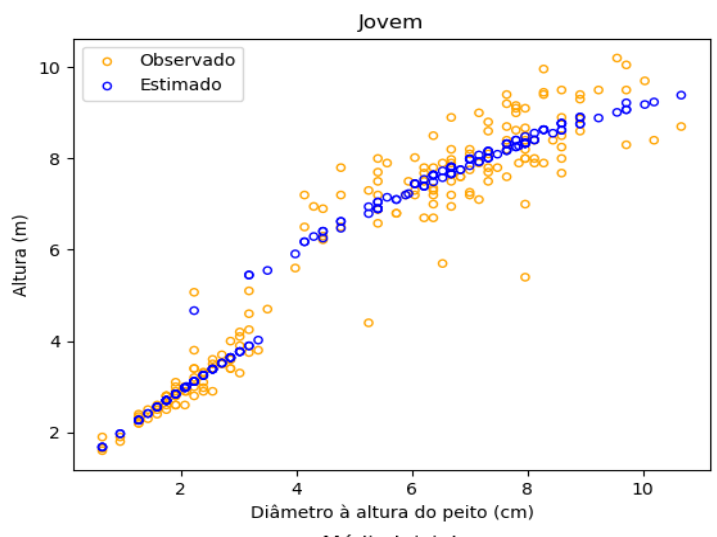

Média Inicial

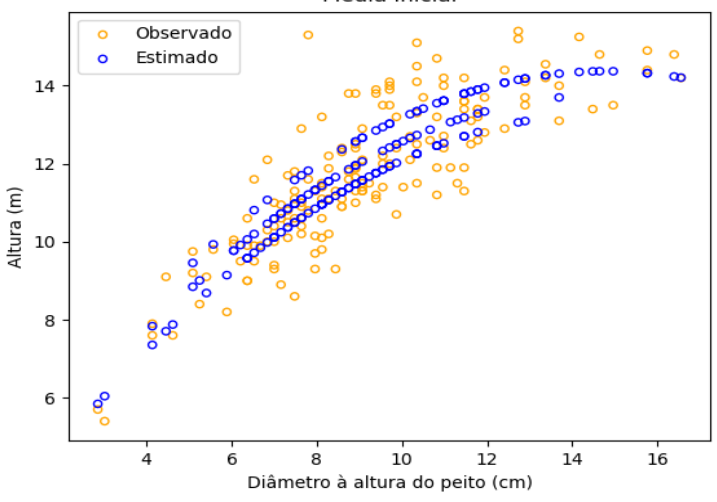

Média Avançada

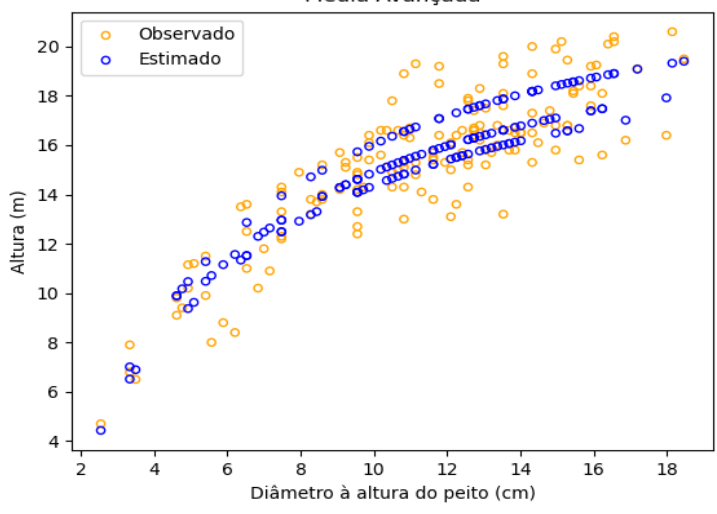

Madura

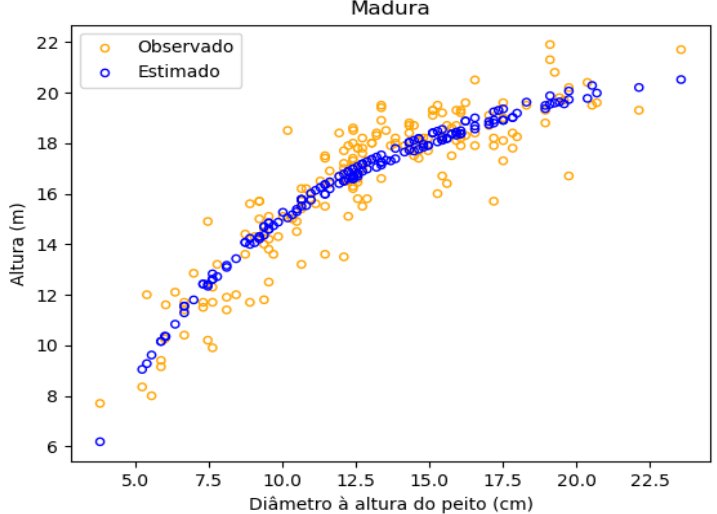

Figura 2. Relação hipsométrica obtida a partir dos melhores resultados em relação à altura total (m) e o diâmetro altura do peito $(\mathrm{cm})$ para indivíduos de acácia-negra agrupados nas classes: Jovem, Médio Inicial com o modelo Parabólico (2), e Médio Avançado e Maduro com o modelo Exponencial (1).

\section{AGRADECIMENTOS}

Os autores agradecem a empresa TANAC S.A./TANAGRO S.A. pela colaboração e fornecimento dos dados para elaboração do presente trabalho.

Ao Centro de Excelência de Pesquisas em Fixação de Carbono na Biomassa pela disponibilidade de equipamentos especializados que foram de suma importância para a execução do projeto.

\section{REFERÊNCIAS}

ABRÃO, S.F. et al. Influência das condições ambientais no crescimento de dois grupos arbóreos em floresta com araucária. BIOFIC scientific jornal, v.2, n.2, p.10-15, 2017.

AZEVEDO, C.P. et al. Relação hipsométrica para quatro espécies florestais em plantio homogêneo e em diferentes idades na Amazônia Ocidental. Boletim de Pesquisa Florestal, n.39, p.5-29, 1999.

BARTOSZECK, A.C.P.S. et al. Dinâmica da relação hipsométrica em função da idade, do sítio e da densidade inicial de povoamentos de bracatinga da região metropolitana de Curitiba, PR. Revista Árvore, n.28. p.517-533, 2004.

BARRETO, T.G. et al. Influência das características químicas do solo na dinâmica de crescimento de um fragmento florestal. Magistra, v.28, n.1, p.1-12, 2016.

BEHLING, A. et al. Critical analyses when modeling tree biomass to ensure additivity of its componentes. Anais da Academia Brasileira de Ciências, v.90, n.2, p.1760-1774, 2018.

CALDEIRA, M.V.W. et al. Relação hipsométrica para Acacia mearnsii com diferentes idades. Boletim de Pesquisa Florestal, n45, p.57-68, 2002.

CASTRO, R.V.O. et al. Modelagem do crescimento e da produção de povoamentos de Eucalyptus em nível de distribuição diamétrica utilizando índice de local. Revista Árvore, v.40, n.1, p.107-116, 2016.

CHAPMAN, H.H.; MEYER, W.H. Forest mensuration. McGraw-Hill Book Company, 1949.

COSTA, E.A. et al. Influência da posição sociológica na relação hipsométrica de Araucaria angustifólia. Revista Brasileira de Ciências Agrárias, v.9, n.1, p.110-116, 2014.

FIGUEIREDO FILHO, A. et al. Crescimento, mortalidade, ingresso e distribuição diamétrica em Floresta Ombrófila Mista. Revista Floresta, v.40, n.4, p.763-776, 2010.

FINGER, C.A.G. Fundamentos de biometria florestal. UFSM, 1992.

GUIMARÃES, D.P. Desenvolvimento de um modelo de distribuição diamétrica de passo invariante para Prognose e projeção da estrutura de povoamentos de eucalipto. 1994. 160p. (Tese de Doutorado). 
HUSCH, B.; BEERS, T.W. Forest measurements. John Wiley \& Son., 2002.

IBÁ - INDÚSTRIA BRASILEIRA DE ÁRVORES. Relatório IBÁ 2020. São Paulo, 2020.

LOETSCH, F. et al. Forest Inventory. Munchen: BLV Verlagsgesellschaft, v.2, 1973.

MACHADO, S.A.; FIGUEIREDO FILHO, A. Dendrometria. Editora UNICENTRO, v.2, 2009.

MÜLLER, M.D. et al. Equações de altura, volume e afilamento para eucalipto e acácia estabelecidos em sistema silvipastoril. Floresta, v.44, n.3, p.473-484, 2014.

PLÁCIDO, A.C. et al. A distribuição diamétrica para bracatingais em diferentes idades, sítios e densidades na região metropolitana de Curitiba. Revista Floresta, v.34, n.3, p.305-323, 2004.

RIBEIRO, A. et al. Strategies and methodologys for adjustment of hypsometric models of Eucalyptus sp. Stands. Cerne, v.16, n.1, p.22-31, 2015

SANQUETTA, C.R. Experiência de monitoramento no bioma Mata Atlântica com uso de parcelas permanentes. Curitiba: MultiGraphic. 2008.

SANQUETTA, C.R. et al. Inventários Florestais: planejamento e execução. 3 ed. Curitiba: Multi-Graphic Gráfica e Editora, 2014a.
SANQUETTA, C.R. et al. Relação hipsométrica em inventários précorte em povoamentos de Acacia mearnsiiDe Wild. Científica, v.42, n.1, p.80-90, 2014b.

SCOLFORO, J.R.S. Biometria florestal: técnicas de regressão aplicada para estimar: volume, biomassa, relação hipsométrica e múltiplos produtos de madeira. Belo Horizonte: CEMIG; Lavras: UFLA: FAEPE, v.2, 1997.

SOARES, T.S. et al. Uso de diferentes alternativas para viabilizar a relação hipsométrica no povoamento florestal. Revista Árvore, v.28, n.6, p.845-854, 2004.

SOARES, G.M. Dinâmica do crescimento em plantios mistos de Eucalyptus globulus e Acacia mearnsii. 2015. 215p. (Tese de Doutorado).

STOLLE, L. et al. Modelos hipsométricos para um povoamento jovem de Khaya ivorensis A. Chev. BIOFIX Scientific Journal, v.3, n.2, p.231-236, 2018.

VIEIRA, M.; SCHUMACHER, M.V. Deposição de serapilheira e de macronutrientes em um povoamento de acácia-negra (Acacia mearnsii De Wild.) no Rio Grande do Sul. Ciência Florestal, v.20, n.2, p.225-233, 2010.

Recebido em 31-03-2021

Aceito em 10-06-2021 


\section{APÊNDICE}

Tabela 4. Parâmetros de cada modelo para cálculo da altura total de indivíduos de acácia-negra.

\begin{tabular}{|c|c|c|c|c|c|c|c|c|c|}
\hline \multirow{2}{*}{ Modelos } & \multirow[b]{2}{*}{ GL } & \multicolumn{4}{|c|}{ Jovem } & \multicolumn{4}{|c|}{ Média Inicial } \\
\hline & & $\beta_{0}$ & $\beta_{1}$ & $\beta_{2}$ & $\beta_{3}$ & $\beta_{0}$ & $\beta_{1}$ & $\boldsymbol{\beta}_{2}$ & $\beta_{3}$ \\
\hline Assmann (1) & 2 & 8,4353 & $-8,0662$ & - & - & 16,2654 & $-37,9016$ & - & - \\
\hline Assmann (2) & 2 & 6,6362 & $-4,1709$ & - & - & 13,2771 & $-96,0955$ & - & - \\
\hline Azevedo et al. (1999) (1) & 2 & 3,2412 & 0,0142 & - & - & 5,3999 & 0,0000 & - & - \\
\hline Azevedo et al. (1999) (2) & 2 & 1,1547 & 0,0156 & - & - & 2,2208 & 0,0025 & - & - \\
\hline Azevedo et al. (1999) (3) & 2 & 0,6036 & 0,2710 & - & - & 0,6029 & 0,2232 & - & - \\
\hline Azevedo et al. (1999) (4) & 2 & 0,6036 & 0,2710 & - & - & 0,6029 & 0,2232 & - & - \\
\hline Azevedo et al. (1999) (5) & 2 & 3,2614 & 0,0813 & - & - & 9,1618 & 0,0285 & - & - \\
\hline Azevedo et al. (2011) (1) & 2 & 1,1401 & 0,0254 & - & - & 1,6462 & 0,0111 & - & - \\
\hline Azevedo et al. (2011) (2) & 3 & 1,6051 & $-0,8957$ & 0,0196 & - & 2,3600 & $-4,0400$ & 0,0085 & - \\
\hline Azevedo et al. (2011) (3) & 3 & 5,0834 & $-3,5095$ & 0,0588 & - & 13,9094 & $-26,8721$ & 0,0115 & - \\
\hline Azevedo et al. (2011) (4) & 3 & 1,7080 & $-1,0657$ & - & - & 2,7862 & $-3,2004$ & - & - \\
\hline Azevedo et al. (2011) (5) & 2 & 0,5847 & 0,8296 & - & - & 1,7262 & 0,5635 & - & - \\
\hline Azevedo et al. (2011) (6) & 2 & 1,2327 & 0,7969 & - & - & 1,6137 & 0,8286 & - & - \\
\hline Backman modificada & 3 & 0,2621 & 4,0444 & $-0,5068$ & - & 0,3263 & 4,7583 & 1,4019 & - \\
\hline Berkhout & 2 & 0,7766 & 0,1757 & - & - & 1,9452 & 0,0550 & - & - \\
\hline Curtis (1967) & 2 & 2,2046 & $-1,7408$ & - & - & 2,8869 & $-3,6720$ & - & - \\
\hline Exponencial (1) & 3 & 0,7176 & $-0,8284$ & 0,8110 & - & 3,2009 & $-3,3490$ & $-0,1275$ & - \\
\hline Exponencial (2) & 2 & 0,7766 & 0,1757 & - & - & 1,9452 & 0,0550 & - & - \\
\hline Exponencial (3) & 2 & 1,1547 & 0,0156 & - & - & 2,2208 & 0,0025 & - & - \\
\hline Exponencial (4) & 2 & 2,2046 & $-1,7408$ & - & - & 2,8869 & $-3,6720$ & - & - \\
\hline Finger (1992) & 2 & 6,6362 & $-4,1709$ & - & - & 13,2771 & $-96,0955$ & - & - \\
\hline Henricksen (1950) & 2 & 0,8112 & 3,5859 & - & - & $-0,1193$ & 5,4573 & - & - \\
\hline Hiperbólico & 3 & $-2,2123$ & $-1,0439$ & 5,6043 & - & 19,0060 & $-84,0683$ & $-2,1420$ & - \\
\hline Linear Simples & 2 & 1,4085 & 0,8946 & - & - & 6,1720 & 0,6079 & - & - \\
\hline Naslung (1) & 2 & 0,6763 & 0,0929 & - & - & 1,3178 & 0,0588 & - & - \\
\hline Naslung (2) & 2 & 1,0473 & 0,2439 & - & - & 0,7275 & 0,2272 & - & - \\
\hline Naslung (Prodan) (1) & 3 & 0,0429 & 0,3228 & 0,06250 & - & 0,3197 & 0,2136 & 0,0482 & - \\
\hline Naslung (Prodan) (2) & 3 & 0,3018 & 0,3836 & 0,06711 & - & 0,5247 & 0,2356 & 0,0516 & - \\
\hline Não Referenciado (1) & 2 & 0,3170 & 0,4286 & - & - & 0,2256 & 0,5827 & - & - \\
\hline Parabólico (1) & 3 & 0,0790 & 1,6222 & $-0,0715$ & - & 2,1947 & 1,4908 & $-0,0454$ & - \\
\hline Parabólico (2) & 4 & $-0,8146$ & 0,9971 & $-0,0326$ & 1,8692 & 6,3283 & 1,5472 & $-0,0523$ & $-1,4550$ \\
\hline Parabólico (3) & 3 & 0,3235 & 0,4237 & $-0,0244$ & - & 1,4439 & 0,1662 & $-0,0057$ & - \\
\hline Petterson & 2 & 0,4504 & 0,7122 & - & - & 0,3788 & 0,6923 & - & - \\
\hline Petterson (2) & 2 & 0,3170 & 0,4286 & - & - & 0,2256 & 0,5827 & - & - \\
\hline Pollanschutz & 2 & 2,1559 & $-2,7553$ & - & - & 2,8333 & $-4,2413$ & - & - \\
\hline Potencial (1) & 3 & 0,2980 & 0,5209 & 0,4196 & - & 1,7031 & 0,4673 & $-0,0977$ & - \\
\hline Potencial (2) & 2 & 0,6194 & 0,3661 & - & - & 1,3433 & 0,2543 & - & - \\
\hline Potencial (3) & 2 & 0,6194 & $-0,7321$ & - & - & 1,3433 & $-0,5086$ & - & - \\
\hline Prodan (1965) (1) & 3 & 0,3018 & 0,3836 & 0,0671 & - & 0,5247 & 0,2356 & 0,0516 & - \\
\hline Prodan (1965) (2) & 3 & 1,5599 & 0,2542 & 0,0857 & - & 0,8959 & 0,2493 & 0,0560 & - \\
\hline Sanquetta et al. (2013) & 3 & 2,3063 & $-1,2942$ & - & - & 11,2759 & $-20,5434$ & - & - \\
\hline Schneider (1986) (1) & 2 & 8,4353 & $-8,0662$ & - & - & 16,2654 & $-37,9016$ & - & - \\
\hline Schneider (1986) (2) & 2 & 0,1085 & 0,8946 & 0,0087 & - & 4,8720 & 0,6079 & 0,0005 & - \\
\hline Schumacher (1986) (1) & 3 & $-1,2210$ & 1,6222 & $-0,0715$ & - & 0,8947 & 1,4908 & $-0,0454$ & - \\
\hline Schumacher (1986) (2) & 3 & 0,2980 & 0,5209 & 0,4196 & - & 1,7031 & 0,4673 & $-0,0977$ & - \\
\hline Schumacher-hall (i) & 3 & 0,7195 & 0,5175 & 0,5780 & - & 1,7061 & 0,4660 & $-0,2673$ & - \\
\hline Semilogarítmico (i) & 3 & $-0,9802$ & 2,4087 & 2,3393 & - & 4,5708 & 4,9194 & $-1,2737$ & - \\
\hline Silva (1980) & 3 & 0,3990 & 0,8276 & 0,2717 & - & 2,2166 & 0,2236 & $-2,1272$ & - \\
\hline Stoffels \& Soest (1953) & 2 & 0,6194 & 0,7321 & - & - & 1,3433 & 0,5086 & - & - \\
\hline Stoffels \& Soest (1953) (2) & 2 & 0,6194 & 1,6857 & - & - & 1,3433 & 1,1711 & - & - \\
\hline Trorey (1932) & 3 & 0,0790 & 1,6222 & $-0,0715$ & - & 2,1947 & 1,4908 & $-0,0454$ & - \\
\hline
\end{tabular}




\begin{tabular}{|c|c|c|c|c|c|c|c|c|c|}
\hline \multirow{2}{*}{ Modelos } & \multirow{2}{*}{ GL } & \multicolumn{4}{|c|}{ Média Avançada } & \multicolumn{4}{|c|}{ Madura } \\
\hline & & $\boldsymbol{\beta}_{0}$ & $\beta_{1}$ & $\boldsymbol{\beta}_{2}$ & $\beta_{3}$ & $\boldsymbol{\beta}_{0}$ & $\beta_{1}$ & $\beta_{2}$ & $\beta_{3}$ \\
\hline Assmann (1) & 2 & 20,4172 & $-50,2357$ & - & - & 23,2962 & $-78,8975$ & - & - \\
\hline Assmann (2) & 2 & 16,9412 & $-125,9117$ & - & - & 19,0623 & $-301,3981$ & - & - \\
\hline Azevedo et al. (1999) (1) & 2 & 6,2551 & 0,0013 & - & - & 6,2544 & 0,0016 & - & - \\
\hline Azevedo et al. (1999) (2) & 2 & 2,3769 & 0,0023 & - & - & 2,5118 & 0,0015 & - & - \\
\hline Azevedo et al. (1999) (3) & 2 & 0,6195 & 0,1976 & - & - & 0,7169 & 0,1878 & - & - \\
\hline Azevedo et al. (1999) (4) & 2 & 0,6195 & 0,1976 & - & - & 0,7169 & 0,1878 & - & - \\
\hline Azevedo et al. (1999) (5) & 2 & 10,8753 & 0,0315 & - & - & 12,3568 & 0,0227 & - & - \\
\hline Azevedo et al. (2011) (1) & 2 & 1,6054 & 0,0089 & - & - & 1,8693 & 0,007 & - & - \\
\hline Azevedo et al. (2011) (2) & 3 & 2,2743 & $-3,811$ & 0,0069 & - & 2,6355 & $-5,6838$ & 0,0055 & - \\
\hline Azevedo et al. (2011) (3) & 3 & 17,0909 & $-35,4126$ & 0,0129 & - & 21,234 & $-65,8594$ & 0,0051 & - \\
\hline Azevedo et al. (2011) (4) & 3 & 3,0428 & $-3,794$ & - & - & 3,2484 & $-5,4647$ & - & - \\
\hline Azevedo et al. (2011) (5) & 2 & 1,7447 & 0,6459 & - & - & 1,8895 & 0,606 & - & - \\
\hline Azevedo et al. (2011) (6) & 2 & 1,5691 & 0,98 & - & - & 1,4302 & 1,0411 & - & - \\
\hline Backman modificada & 3 & $-0,4032$ & 5,578 & 2,947 & - & $-1,1259$ & 1,7117 & 14,6285 & - \\
\hline Berkhout & 2 & 2,0704 & 0,0559 & - & - & 2,2167 & 0,0439 & - & - \\
\hline Curtis (1967) & 2 & 3,1296 & $-4,1808$ & - & - & 3,2647 & $-5,5675$ & - & - \\
\hline Exponencial (1) & 3 & 2,341 & $-4,1524$ & 0,1471 & - & 3,0048 & $-5,5757$ & 0,0254 & - \\
\hline Exponencial (2) & 2 & 2,0704 & 0,0559 & - & - & 2,2167 & 0,0439 & - & - \\
\hline Exponencial (3) & 2 & 2,3769 & 0,0023 & - & - & 2,5118 & 0,0015 & - & - \\
\hline Exponencial (4) & 2 & 3,1296 & $-4,1808$ & - & - & 3,2647 & $-5,5675$ & - & - \\
\hline Finger (1992) & 2 & 16,9412 & $-125,912$ & - & - & 19,0623 & $-301,3981$ & - & - \\
\hline Henricksen (1950) & 2 & $-1,3424$ & 7,0279 & - & - & $-3,5357$ & 7,9793 & - & - \\
\hline Hiperbólico (1) & 3 & 1,2989 & $-126,0816$ & 2,928 & - & 16,0883 & $-301,6136$ & 0,2903 & - \\
\hline Linear Simples & 2 & 6,9889 & 0,733 & - & - & 8,0494 & 0,6524 & - & - \\
\hline Naslung (1) & 2 & 1,3764 & 0,0472 & - & - & 1,7632 & 0,0435 & - & - \\
\hline Naslung (2) & 2 & 0,7285 & 0,1995 & - & - & 0,8244 & 0,1897 & - & - \\
\hline Naslung (Prodan) (1) & 3 & $-0,0207$ & 0,2804 & 0,0345 & - & 1,2016 & 0,0909 & 0,0402 & - \\
\hline Naslung (Prodan) (2) & 3 & 0,143 & 0,3 & 0,0364 & - & 1,5904 & 0,0774 & 0,043 & - \\
\hline Não Referenciado (1) & 2 & 0,1971 & 0,6246 & - & - & 0,185 & 0,7523 & - & - \\
\hline Parabolico (3) & 3 & 1,5247 & 0,171 & $-0,0054$ & - & 1,6107 & 0,1459 & $-0,0039$ & - \\
\hline Parabólico (1) & 3 & 2,4463 & 1,6917 & $-0,045$ & - & 1,0735 & 1,8268 & $-0,0451$ & - \\
\hline Parabólico (2) & 4 & $-11,065$ & 1,9049 & $-0,0564$ & 2,376 & $-3,0917$ & 1,7957 & $-0,0437$ & 0,422 \\
\hline Petterson & 2 & 0,3451 & 0,7438 & - & - & 0,3312 & 0,8894 & - & - \\
\hline Petterson (2) & 2 & 0,1971 & 0,6246 & - & - & 0,185 & 0,7523 & - & - \\
\hline Pollanschutz & 2 & 3,0938 & $-4,7582$ & - & - & 3,2302 & $-6,1633$ & - & - \\
\hline Potencial (1) & 3 & 0,8138 & 0,5495 & 0,1102 & - & 1,0163 & 0,5513 & 0,0375 & - \\
\hline Potencial (2) & 2 & 1,3841 & 0,2787 & - & - & 1,4066 & 0,2745 & - & - \\
\hline Potencial (3) & 2 & 1,3841 & $-0,5573$ & - & - & 1,4066 & $-0,549$ & - & - \\
\hline Prodan (1965) (1) & 3 & 0,143 & 0,3 & 0,0364 & - & 1,5904 & 0,0774 & 0,043 & - \\
\hline Prodan (1965) (2) & 3 & 0,4532 & 0,3083 & 0,0391 & - & 2,1633 & 0,0489 & 0,0467 & - \\
\hline Sanquetta et al. (2013) & 3 & 13,8637 & $-27,8213$ & - & - & 18,5524 & $-56,1848$ & - & - \\
\hline Schneider (1986) (1) & 2 & 20,4172 & $-50,2357$ & - & - & 23,2962 & $-78,8975$ & - & - \\
\hline Schneider (1986) (2) & 2 & 5,6889 & 0,733 & 0,0003 & - & 6,7494 & 0,6524 & $4,06 \mathrm{E}-05$ & - \\
\hline Schumacher (1986) (1) & 3 & 1,1463 & 1,6917 & $-0,045$ & - & $-0,2265$ & 1,8268 & $-0,0451$ & - \\
\hline Schumacher (1986) (2) & 3 & 0,8138 & 0,5495 & 0,1102 & - & 1,0163 & 0,5513 & 0,0375 & - \\
\hline Schumacher-hall (i) & 3 & 0,424 & 0,5498 & 0,5841 & - & 0,5038 & 0,5514 & 0,3854 & - \\
\hline Semilogarítmico (i) & 3 & $-12,5047$ & 6,8762 & 2,1564 & - & $-9,3592$ & 8,0148 & 0,5595 & - \\
\hline Silva (1980) & 3 & 2,6367 & 0,1595 & $-3,0505$ & - & 2,8923 & 0,1113 & $-4,4878$ & - \\
\hline Stoffels \& Soest (1953) & 2 & 1,3841 & 0,5573 & - & - & 1,4066 & 0,549 & - & - \\
\hline Stoffels \& Soest (1953) (2) & 2 & 1,3841 & 1,2832 & - & - & 1,4066 & 1,264 & - & - \\
\hline Trorey (1932) & 3 & 2,4463 & 1,6917 & $-0,045$ & - & 1,0735 & 1,8268 & $-0,0451$ & - \\
\hline
\end{tabular}

Em que: $d=$ diâmetro altura do peito $(\mathrm{cm}) ; \mathrm{h}=$ altura total $(\mathrm{m}) ; \mathrm{GL}=$ Grau de liberdade; $\beta_{0}, \beta_{1}, \beta_{2}$ e $\beta_{3}$, = parâmetros; 
Tabela 5. Viés, precisão e acurácia para os modelos para cálculo da altura total de indivíduos de acácia-negra.

\begin{tabular}{|c|c|c|c|c|c|c|}
\hline \multirow{2}{*}{ Modelo } & \multicolumn{6}{|c|}{ Jovem } \\
\hline & $\overline{\boldsymbol{e}}$ & $\overline{\boldsymbol{e}}_{\%}$ & $S_{e}$ & $\boldsymbol{S}_{\boldsymbol{e} \%}$ & $m_{x}$ & $\boldsymbol{m}_{\boldsymbol{x} \%}$ \\
\hline Assmann (1) & $1.87275 \mathrm{E}-15$ & $3.57426 \mathrm{E}-14$ & 1.57640276 & 30.0866927 & 1.57640276 & 30.0866927 \\
\hline Assmann (2) & 0.535375973 & 10.21800569 & 1.908486313 & 36.42472768 & 1.982157269 & 37.83078676 \\
\hline Azevedo et al. (1999) (1) & 0.513643215 & 9.803221583 & 0.94944931 & 18.12087009 & 1.079482906 & 20.60264756 \\
\hline Azevedo et al. (1999) (2) & -0.106034567 & -2.023740073 & 1.766241091 & 33.70988323 & 1.769421069 & 33.77057523 \\
\hline Azevedo et al. (1999) (3) & 0.014253958 & 0.272046239 & 0.594393013 & 11.34438507 & 0.594563898 & 11.34764653 \\
\hline Azevedo et al. (1999) (4) & 0.014253958 & 0.272046239 & 0.594393013 & 11.34438507 & 0.594563898 & 11.34764653 \\
\hline Azevedo et al. (1999) (5) & $-1.26686 \mathrm{E}-15$ & $-2.41788 \mathrm{E}-14$ & 1.039205063 & 19.83391821 & 1.039205063 & 19.83391821 \\
\hline Azevedo et al. (2011) (1) & -0.193395072 & -3.691073281 & 0.984981701 & 18.79902935 & 1.003788127 & 19.15796248 \\
\hline Azevedo et al. (2011) (2) & -0.089470031 & -1.707594916 & 0.917448771 & 17.51011857 & 0.921801027 & 17.59318427 \\
\hline Azevedo et al. (2011) (3) & $7.4669 \mathrm{E}-15$ & $1.42511 \mathrm{E}-13$ & 0.855538622 & 16.3285223 & 0.855538622 & 16.3285223 \\
\hline Azevedo et al. (2011) (4) & 1.583318909 & 30.21869201 & 1.944119182 & 37.10480464 & 2.507289007 & 47.85327443 \\
\hline Azevedo et al. (2011) (5) & 0.01527717 & 0.291574923 & 0.643819673 & 12.28772567 & 0.644000903 & 12.29118456 \\
\hline Azevedo et al. (2011) (6) & 0.019909473 & 0.379985503 & 0.611504808 & 11.67097504 & 0.611828831 & 11.67715922 \\
\hline Backman modificada & $1.19801 \mathrm{E}-15$ & $2.28648 \mathrm{E}-14$ & 0.749640206 & 14.30738077 & 0.749640206 & 14.30738077 \\
\hline Berkhout & -0.059159775 & -1.129103557 & 1.136433871 & 21.68959452 & 1.137972681 & 21.71896372 \\
\hline Curtis (1967) & 0.091419751 & 1.744806614 & 1.161744853 & 22.17267141 & 1.165336293 & 22.24121641 \\
\hline Exponencial (1) & 0.007135175 & 0.136179555 & 0.606057175 & 11.56700335 & 0.606099176 & 11.56780495 \\
\hline Exponencial (2) & 0.011787967 & 0.224981173 & 1.121246485 & 21.39973319 & 1.121308449 & 21.4009158 \\
\hline Exponencial (3) & -0.106034567 & -2.023740073 & 1.766241091 & 33.70988323 & 1.769421069 & 33.77057523 \\
\hline Exponencial (4) & 0.091419751 & 1.744806614 & 1.161744853 & 22.17267141 & 1.165336293 & 22.24121641 \\
\hline Finger (1992) & $-4.13106 E-16$ & $-7.88441 E-15$ & 2.201559649 & 42.01822676 & 2.201559649 & 42.01822676 \\
\hline Henricksen (1950) & $-4.27565 \mathrm{E}-15$ & $-8.16036 \mathrm{E}-14$ & 0.764849232 & 14.59765512 & 0.764849232 & 14.59765512 \\
\hline Hiperbólico (1) & $-9.31555 E-15$ & $-1.77793 \mathrm{E}-13$ & 0.730886328 & 13.94945056 & 0.730886328 & 13.94945056 \\
\hline Hiperbólico (2) & -3.362114326 & -64.16818288 & 3.032905022 & 57.88500485 & 4.527949383 & 86.41891853 \\
\hline Linear Simples & $-3.75927 E-15$ & $-7.17481 E-14$ & 0.67342717 & 12.8528044 & 0.67342717 & 12.8528044 \\
\hline Não Referenciado (1) & 0.414946907 & 7.919537065 & 1.121705132 & 21.40848676 & 1.195994707 & 22.8263526 \\
\hline Naslung (1) & 0.796797721 & 15.20741322 & 0.639135168 & 12.19831878 & 1.021459921 & 19.49524039 \\
\hline Naslung (2) & 0.051865278 & 0.98988325 & 0.554434934 & 10.58175862 & 0.55685555 & 10.62795767 \\
\hline Naslung (Prodan) (1) & 0.834980823 & 15.93616307 & 0.638722476 & 12.19044228 & 1.051265607 & 20.06410168 \\
\hline Naslung (Prodan) (2) & 0.050990937 & 0.97319587 & 0.582798818 & 11.12310216 & 0.585025246 & 11.16559501 \\
\hline Parabólico (1) & $-2.3251 \mathrm{E}-14$ & $-4.43761 \mathrm{E}-13$ & 0.543736796 & 10.37757755 & 0.543736796 & 10.37757755 \\
\hline Parabólico (2) & $1.02936 \mathrm{E}-13$ & $1.9646 \mathrm{E}-12$ & 0.470977773 & 8.988923318 & 0.470977773 & 8.988923318 \\
\hline Parabólico (3) & -0.002540507 & -0.048487253 & 0.54837631 & 10.46612575 & 0.548382195 & 10.46623807 \\
\hline Petterson & 0.372633197 & 7.111951823 & 0.838822953 & 16.00949267 & 0.917866791 & 17.51809677 \\
\hline Petterson (2) & 0.414946907 & 7.919537065 & 1.121705132 & 21.40848676 & 1.195994707 & 22.8263526 \\
\hline Pollanschutz & 0.33962513 & 6.481970974 & 1.028973837 & 19.63864846 & 1.083573895 & 20.68072681 \\
\hline Potencial (1) & -0.003496756 & -0.066737906 & 0.490709497 & 9.365516365 & 0.490721956 & 9.365754146 \\
\hline Potencial (2) & 0.040821686 & 0.779108972 & 0.612946209 & 11.69848512 & 0.614304049 & 11.7244004 \\
\hline Potencial (3) & 0.040821686 & 0.779108972 & 0.612946209 & 11.69848512 & 0.614304049 & 11.7244004 \\
\hline Prodan (1965) (1) & 0.050990937 & 0.97319587 & 0.582798818 & 11.12310216 & 0.585025246 & 11.16559501 \\
\hline Prodan (1965) (2) & 0.049364837 & 0.942160679 & 0.529323274 & 10.10248592 & 0.53162018 & 10.14632389 \\
\hline Sanquetta et al. (2013) & $-9.39472 E-15$ & $-1.79305 E-13$ & 0.656748818 & 12.53448699 & 0.656748818 & 12.53448699 \\
\hline Schneider (1986) (1) & $1.87275 \mathrm{E}-15$ & $3.57426 \mathrm{E}-14$ & 1.57640276 & 30.0866927 & 1.57640276 & 30.0866927 \\
\hline Schneider (1986) (2) & $-4.12762 \mathrm{E}-15$ & $-7.87784 \mathrm{E}-14$ & 0.67342717 & 12.8528044 & 0.67342717 & 12.8528044 \\
\hline Schumacher (1986) (1) & $-1.75605 E-14$ & $-3.35153 \mathrm{E}-13$ & 0.543736796 & 10.37757755 & 0.543736796 & 10.37757755 \\
\hline Schumacher (1986) (2) & -0.003496756 & -0.066737906 & 0.490709497 & 9.365516365 & 0.490721956 & 9.365754146 \\
\hline Schumacher-hall (i) & -0.003496756 & -0.066737906 & 0.490709497 & 9.365516365 & 0.490721956 & 9.365754146 \\
\hline Semilogarítmico (i) & $4.22745 \mathrm{E}-15$ & $8.06838 \mathrm{E}-14$ & 0.532258062 & 10.1584983 & 0.532258062 & 10.1584983 \\
\hline Silva (1980) & 2.064229648 & 39.39719256 & 2.42557275 & 46.29366545 & 3.185034883 & 60.7885042 \\
\hline Stoffels \& Soest (1953) & 0.010369538 & 0.197909513 & 0.612372495 & 11.68753542 & 0.612460285 & 11.68921094 \\
\hline Stoffels \& Soest (1953) (2) & 0.040821686 & 0.779108972 & 0.612946209 & 11.69848512 & 0.614304049 & 11.7244004 \\
\hline
\end{tabular}




\begin{tabular}{|c|c|c|c|c|c|c|}
\hline \multirow{2}{*}{ Modelo } & \multicolumn{6}{|c|}{ Média Inicial } \\
\hline & $\overline{\boldsymbol{e}}$ & $\overline{\boldsymbol{e}}_{\%}$ & $S_{e}$ & $\boldsymbol{S}_{\boldsymbol{e} \%}$ & $m_{x}$ & $\boldsymbol{m}_{x \%}$ \\
\hline Trorey (1932) & $-2.3251 \mathrm{E}-14$ & $-4.43761 \mathrm{E}-13$ & 0.543736796 & 10.37757755 & 0.543736796 & 10.37757755 \\
\hline Assmann (1) & $8.24 \mathrm{E}-15$ & $7.02 \mathrm{E}-14$ & 1.083912 & 9.227143 & 1.083912 & 9.227143 \\
\hline Assmann (2) & 9.925772 & 84.49627 & 2.646564 & 22.52971 & 10.27255 & 87.44831 \\
\hline Azevedo et al. (1999) (1) & 0.004007 & 0.034107 & 1.00227 & 8.532141 & 1.002278 & 8.53221 \\
\hline Azevedo et al. (1999) (2) & -0.01657 & -0.14108 & 1.339511 & 11.40301 & 1.339614 & 11.40388 \\
\hline Azevedo et al. (1999) (3) & 0.060573 & 0.515647 & 0.999529 & 8.508808 & 1.001363 & 8.524418 \\
\hline Azevedo et al. (1999) (4) & 0.060573 & 0.515647 & 0.999529 & 8.508808 & 1.001363 & 8.524418 \\
\hline Azevedo et al. (1999) (5) & $3.07 E-15$ & $2.61 \mathrm{E}-14$ & 1.235564 & 10.51813 & 1.235564 & 10.51813 \\
\hline Azevedo et al. (2011) (1) & -0.00812 & -0.06911 & 1.277464 & 10.87481 & 1.277489 & 10.87503 \\
\hline Azevedo et al. (2011) (2) & 0.055276 & 0.470557 & 1.04673 & 8.910618 & 1.048188 & 8.923034 \\
\hline Azevedo et al. (2011) (3) & $2.8 \mathrm{E}-14$ & $2.39 \mathrm{E}-13$ & 1.019934 & 8.682511 & 1.019934 & 8.682511 \\
\hline Azevedo et al. (2011) (4) & 0.54265 & 4.619479 & 1.064024 & 9.057841 & 1.19441 & 10.1678 \\
\hline Azevedo et al. (2011) (5) & 0.023612 & 0.201001 & 1.058776 & 9.013165 & 1.059039 & 9.015406 \\
\hline Azevedo et al. (2011) (6) & 0.021342 & 0.181679 & 1.0123 & 8.617527 & 1.012525 & 8.619442 \\
\hline Backman modificada & $8.53 \mathrm{E}-13$ & $7.26 \mathrm{E}-12$ & 1.001638 & 8.526757 & 1.001638 & 8.526757 \\
\hline Berkhout & -0.01334 & -0.1136 & 1.182107 & 10.06306 & 1.182182 & 10.0637 \\
\hline Curtis (1967) & 0.003372 & 0.028708 & 1.01458 & 8.636931 & 1.014585 & 8.636978 \\
\hline Exponencial (1) & 0.00087 & 0.007405 & 0.928923 & 7.907753 & 0.928924 & 7.907757 \\
\hline Exponencial (2) & 0.049284 & 0.419543 & 1.180476 & 10.04917 & 1.181504 & 10.05793 \\
\hline Exponencial (3) & -0.01657 & -0.14108 & 1.339511 & 11.40301 & 1.339614 & 11.40388 \\
\hline Exponencial (4) & 0.003372 & 0.028708 & 1.01458 & 8.636931 & 1.014585 & 8.636978 \\
\hline Finger (1992) & $-1.3 \mathrm{E}-15$ & $-1.1 \mathrm{E}-14$ & 1.304455 & 11.10458 & 1.304455 & 11.10458 \\
\hline Henricksen (1950) & $1.27 \mathrm{E}-14$ & $1.08 \mathrm{E}-13$ & 1.002103 & 8.53072 & 1.002103 & 8.53072 \\
\hline Hiperbólico (1) & $-4.2 E-14$ & $-3.6 \mathrm{E}-13$ & 1.132898 & 9.644152 & 1.132898 & 9.644152 \\
\hline Hiperbólico (2) & -5.51074 & -46.9119 & 3.463172 & 29.48135 & 6.508593 & 55.40645 \\
\hline Linear Simples & $6.1 \mathrm{E}-15$ & 5.19E-14 & 1.089382 & 9.27371 & 1.089382 & 9.27371 \\
\hline Não Referenciado (1) & 0.066777 & 0.568462 & 1.000743 & 8.519137 & 1.002968 & 8.538082 \\
\hline Naslung (1) & 1.076132 & 9.160915 & 1.163228 & 9.902343 & 1.584664 & 13.48995 \\
\hline Naslung (2) & 0.069403 & 0.590812 & 0.998869 & 8.503187 & 1.001277 & 8.523688 \\
\hline Naslung (Prodan) (1) & 1.081372 & 9.205524 & 1.128757 & 9.608899 & 1.563156 & 13.30686 \\
\hline Naslung (Prodan) (2) & 0.08317 & 0.708013 & 1.001377 & 8.524542 & 1.004825 & 8.553893 \\
\hline Parabólico (1) & $4.05 \mathrm{E}-13$ & $3.44 \mathrm{E}-12$ & 0.996177 & 8.480271 & 0.996177 & 8.480271 \\
\hline Parabólico (2) & $2.31 \mathrm{E}-13$ & $1.96 \mathrm{E}-12$ & 0.911557 & 7.759916 & 0.911557 & 7.759916 \\
\hline Parabólico (3) & -0.00372 & -0.03166 & 1.007781 & 8.579053 & 1.007788 & 8.579112 \\
\hline Petterson & 0.0657 & 0.559292 & 0.999976 & 8.512612 & 1.002132 & 8.530966 \\
\hline Petterson (2) & 0.066777 & 0.568462 & 1.000743 & 8.519137 & 1.002968 & 8.538082 \\
\hline Pollanschutz & 0.052614 & 0.447891 & 1.009147 & 8.590685 & 1.010518 & 8.602352 \\
\hline Potencial (1) & -0.00799 & -0.06798 & 0.997232 & 8.489255 & 0.997264 & 8.489527 \\
\hline Potencial (2) & 0.039293 & 0.334492 & 1.036902 & 8.826959 & 1.037647 & 8.833294 \\
\hline Potencial (3) & 0.039293 & 0.334492 & 1.036902 & 8.826959 & 1.037647 & 8.833294 \\
\hline Prodan (1965) (1) & 0.08317 & 0.708013 & 1.001377 & 8.524542 & 1.004825 & 8.553893 \\
\hline Prodan (1965) (2) & 0.091584 & 0.77964 & 0.999793 & 8.511053 & 1.003979 & 8.546687 \\
\hline Sanquetta et al. (2013) & $8.24 \mathrm{E}-14$ & $7.02 \mathrm{E}-13$ & 1.009125 & 8.590498 & 1.009125 & 8.590498 \\
\hline Schneider (1986) (1) & $8.24 \mathrm{E}-15$ & $7.02 \mathrm{E}-14$ & 1.083912 & 9.227143 & 1.083912 & 9.227143 \\
\hline Schneider (1986) (2) & $1.05 \mathrm{E}-14$ & 8.95E-14 & 1.089382 & 9.27371 & 1.089382 & 9.27371 \\
\hline Schumacher (1986) (1) & $3.46 \mathrm{E}-13$ & $2.94 \mathrm{E}-12$ & 0.996177 & 8.480271 & 0.996177 & 8.480271 \\
\hline Schumacher (1986) (2) & -0.00799 & -0.06798 & 0.997232 & 8.489255 & 0.997264 & 8.489527 \\
\hline Schumacher-hall (i) & -0.00806 & -0.06864 & 0.995351 & 8.473241 & 0.995384 & 8.473519 \\
\hline Semilogarítmico (i) & $-3.2 \mathrm{E}-13$ & $-2.7 \mathrm{E}-12$ & 0.933406 & 7.945918 & 0.933406 & 7.945918 \\
\hline Silva (1980) & -9.73708 & -82.89 & 2.13483 & 18.17342 & 9.968361 & 84.85883 \\
\hline Stoffels \& Soest (1953) & -0.00653 & -0.05559 & 1.037566 & 8.832607 & 1.037586 & 8.832782 \\
\hline Stoffels \& Soest (1953) (2) & 0.039293 & 0.334492 & 1.036902 & 8.826959 & 1.037647 & 8.833294 \\
\hline Trorey (1932) & $4.05 \mathrm{E}-13$ & 3.44E-12 & 0.996177 & 8.480271 & 0.996177 & 8.480271 \\
\hline
\end{tabular}




\begin{tabular}{|c|c|c|c|c|c|c|}
\hline \multirow{2}{*}{ Modelo } & \multicolumn{6}{|c|}{ Média Avançada } \\
\hline & $\overline{\boldsymbol{e}}$ & $\overline{\boldsymbol{e}}_{\%}$ & $S_{e}$ & $\boldsymbol{S}_{\boldsymbol{e} \%}$ & $m_{x}$ & $\boldsymbol{m}_{\boldsymbol{x} \%}$ \\
\hline Assmann (1) & $1,92729 \mathrm{E}-15$ & $1,25776 \mathrm{E}-14$ & 1,5492 & 10,11 & 1,5492 & 10,11 \\
\hline Assmann (2) & 11,14969482 & 72,76347579 & 4,1478 & 27,07 & 11,8962 & 77,64 \\
\hline Azevedo et al. (1999) (1) & 0,020200986 & 0,131832664 & 1,4229 & 9,29 & 1,4231 & 9,29 \\
\hline Azevedo et al. (1999) (2) & $-0,058340135$ & $-0,380730692$ & 2,0179 & 13,17 & 2,0187 & 13,17 \\
\hline Azevedo et al. (1999) (3) & 0,09929908 & 0,648030852 & 1,4078 & 9,19 & 1,4113 & 9,21 \\
\hline Azevedo et al. (1999) (4) & 0,09929908 & 0,648030852 & 1,4078 & 9,19 & 1,4113 & 9,21 \\
\hline Azevedo et al. (1999) (5) & $-2,42653 E-15$ & $-1,58356 \mathrm{E}-14$ & 1,8204 & 11,88 & 1,8204 & 11,88 \\
\hline Azevedo et al. (2011) (1) & $-0,039872313$ & $-0,260208743$ & 1,8637 & 12,16 & 1,8642 & 12,17 \\
\hline Azevedo et al. (2011) (2) & 0,0868952 & 0,5670825 & 1,5564 & 10,16 & 1,5588 & 10,17 \\
\hline Azevedo et al. (2011) (3) & $5,04114 \mathrm{E}-14$ & $3,28987 \mathrm{E}-13$ & 1,4231 & 9,29 & 1,4231 & 9,29 \\
\hline Azevedo et al. (2011) (4) & 0,760930478 & 4,965871019 & 1,4757 & 9,63 & 1,6603 & 10,84 \\
\hline Azevedo et al. (2011) (5) & 0,03997311 & 0,260866551 & 1,5220 & 9,93 & 1,5225 & 9,94 \\
\hline Azevedo et al. (2011) (6) & 0,034216992 & 0,223301829 & 1,4340 & 9,36 & 1,4344 & 9,36 \\
\hline Backman modificada & $-2,77715 E-14$ & $-1,81238 \mathrm{E}-13$ & 1,4070 & 9,18 & 1,4070 & 9,18 \\
\hline Berkhout & $-0,046388237$ & $-0,302731994$ & 1,7583 & 11,47 & 1,7589 & 11,48 \\
\hline Curtis (1967) & 0,002316303 & 0,015116313 & 1,4219 & 9,28 & 1,4219 & 9,28 \\
\hline Exponencial (1) & $-0,000862784$ & $-0,005630571$ & 1,2038 & 7,86 & 1,2038 & 7,86 \\
\hline Exponencial (2) & 0,084974627 & 0,554548737 & 1,7526 & 11,44 & 1,7547 & 11,45 \\
\hline Exponencial (3) & $-0,058340135$ & $-0,380730692$ & 2,0179 & 13,17 & 2,0187 & 13,17 \\
\hline Exponencial (4) & 0,002316303 & 0,015116313 & 1,4219 & 9,28 & 1,4219 & 9,28 \\
\hline Finger (1992) & $1,92729 E-15$ & $1,25776 \mathrm{E}-14$ & 1,9353 & 12,63 & 1,9353 & 12,63 \\
\hline Henricksen (1950) & 1,15289E-14 & $7,52382 E-14$ & 1,4093 & 9,20 & 1,4093 & 9,20 \\
\hline Hiperbólico (1) & $-5,71755 E-13$ & $-3,7313 \mathrm{E}-12$ & 1,7216 & 11,24 & 1,7216 & 11,24 \\
\hline Linear Simples & $-1,23648 \mathrm{E}-14$ & $-8,06936 \mathrm{E}-14$ & 1,5719 & 10,26 & 1,5719 & 10,26 \\
\hline Não Referenciado (1) & 0,092325021 & 0,602517786 & 1,4081 & 9,19 & 1,4111 & 9,21 \\
\hline Naslung (1) & 1,362619471 & 8,892524009 & 1,6355 & 10,67 & 2,1287 & 13,89 \\
\hline Naslung (2) & 0,114054125 & 0,744323027 & 1,4098 & 9,20 & 1,4144 & 9,23 \\
\hline Naslung (Prodan) (1) & 1,379491635 & 9,002632609 & 1,5797 & 10,31 & 2,0973 & 13,69 \\
\hline Naslung (Prodan) (2) & 0,130356018 & 0,850710008 & 1,4111 & 9,21 & 1,4171 & 9,25 \\
\hline Parabólico (1) & $-3,8937 E-13$ & $-2,54105 \mathrm{E}-12$ & 1,4315 & 9,34 & 1,4315 & 9,34 \\
\hline Parabólico (2) & $9,36813 \mathrm{E}-13$ & $6,11369 \mathrm{E}-12$ & 1,2537 & 8,18 & 1,2537 & 8,18 \\
\hline Parabolico (3) & $-0,018271459$ & $-0,119240471$ & 1,5002 & 9,79 & 1,5003 & 9,79 \\
\hline Petterson & 0,08807519 & 0,57478317 & 1,4089 & 9,19 & 1,4117 & 9,21 \\
\hline Petterson (2) & 0,092325021 & 0,602517786 & 1,4081 & 9,19 & 1,4111 & 9,21 \\
\hline Pollanschutz & 0,078630858 & 0,513148978 & 1,4153 & 9,24 & 1,4174 & 9,25 \\
\hline Potencial (1) & $-0,028045775$ & $-0,183028152$ & 1,3708 & 8,95 & 1,3710 & 8,95 \\
\hline Potencial (2) & 0,059109799 & 0,385753555 & 1,4923 & 9,74 & 1,4935 & 9,75 \\
\hline Potencial (3) & 0,059109799 & 0,385753555 & 1,4923 & 9,74 & 1,4935 & 9,75 \\
\hline Prodan (1965) (1) & 0,130356018 & 0,850710008 & 1,4111 & 9,21 & 1,4171 & 9,25 \\
\hline Prodan (1965) (2) & 0,148147376 & 0,966817313 & 1,4109 & 9,21 & 1,4186 & 9,26 \\
\hline Sanquetta et al. (2013) & $1,88781 \mathrm{E}-14$ & $1,232 \mathrm{E}-13$ & 1,4129 & 9,22 & 1,4129 & 9,22 \\
\hline Schneider (1986) (1) & $1,92729 \mathrm{E}-15$ & $1,25776 \mathrm{E}-14$ & 1,5492 & 10,11 & 1,5492 & 10,11 \\
\hline Schneider (1986) (2) & $-1,38045 E-14$ & $-9,00889 E-14$ & 1,5719 & 10,26 & 1,5719 & 10,26 \\
\hline Schumacher (1986) (1) & $-3,50047 E-13$ & $-2,28442 \mathrm{E}-12$ & 1,4315 & 9,34 & 1,4315 & 9,34 \\
\hline Schumacher (1986) (2) & $-0,028045775$ & $-0,183028152$ & 1,3708 & 8,95 & 1,3710 & 8,95 \\
\hline Schumacher-hall (i) & $-0,027991836$ & $-0,182676147$ & 1,3734 & 8,96 & 1,3737 & 8,96 \\
\hline Semilogarítmico (i) & $9,32309 E-13$ & $6,08429 \mathrm{E}-12$ & 1,2511 & 8,16 & 1,2511 & 8,16 \\
\hline Silva (1980) & 5,922860276 & 38,65288755 & 1,8927 & 12,35 & 6,2179 & 40,58 \\
\hline Stoffels \& Soest (1953) & $-0,025033288$ & $-0,16336851$ & 1,4946 & 9,75 & 1,4948 & 9,76 \\
\hline Stoffels \& Soest (1953) (2) & 0,059109799 & 0,385753555 & 1,4923 & 9,74 & 1,4935 & 9,75 \\
\hline Trorey (1932) & $-3,8937 \mathrm{E}-13$ & $-2,54105 \mathrm{E}-12$ & 1,4315 & 9,34 & 1,4315 & 9,34 \\
\hline Assmann (1) & $3,36985 \mathrm{E}-15$ & $2,04 \mathrm{E}-14$ & 1,2642 & 7,67 & 1,2642 & 7,67 \\
\hline Assmann (2) & 23,4547 & 142,32 & 7,5197 & 45,63 & 24,6307 & 149,45 \\
\hline
\end{tabular}




\begin{tabular}{|c|c|c|c|c|c|c|}
\hline \multirow{2}{*}{ Modelo } & \multicolumn{6}{|c|}{ Madura } \\
\hline & $\overline{\boldsymbol{e}}$ & $\overline{\boldsymbol{e}}_{\%}$ & $S_{e}$ & $\boldsymbol{S}_{\boldsymbol{e} \%}$ & $m_{x}$ & $\boldsymbol{m}_{\boldsymbol{x} \%}$ \\
\hline Azevedo et al, (1999) (1) & 0,0304 & 0,18 & 1,2948 & 7,86 & 1,2951 & 7,86 \\
\hline Azevedo et al, (1999) (2) & $-0,0394$ & $-0,24$ & 1,9817 & 12,02 & 1,9821 & 12,03 \\
\hline Azevedo et al, (1999) (3) & 0,0725 & 0,44 & 1,1865 & 7,20 & 1,1887 & 7,21 \\
\hline Azevedo et al, (1999) (4) & 0,0725 & 0,44 & 1,1865 & 7,20 & 1,1887 & 7,21 \\
\hline Azevedo et al, (1999) (5) & $4,40432 E-15$ & $2,67 \mathrm{E}-14$ & 1,7535 & 10,64 & 1,7535 & 10,64 \\
\hline Azevedo et al, (2011) (1) & $-0,0359$ & $-0,22$ & 1,5052 & 9,13 & 1,5056 & 9,14 \\
\hline Azevedo et al, (2011) (2) & 0,0621 & 0,38 & 1,1894 & 7,22 & 1,1910 & 7,23 \\
\hline Azevedo et al, (2011) (3) & $-3,2586 \mathrm{E}-14$ & $-1,98 \mathrm{E}-13$ & 1,2276 & 7,45 & 1,2276 & 7,45 \\
\hline Azevedo et al, (2011) (4) & 0,1278 & 0,78 & 1,1794 & 7,16 & 1,1863 & 7,20 \\
\hline Azevedo et al, (2011) (5) & 0,0308 & 0,19 & 1,3861 & 8,41 & 1,3864 & 8,41 \\
\hline Azevedo et al, (2011) (6) & 0,0254 & 0,15 & 1,2609 & 7,65 & 1,2611 & 7,65 \\
\hline Backman modificada & $-8,5653 \mathrm{E}-13$ & $-5,20 \mathrm{E}-12$ & 1,1957 & 7,26 & 1,1957 & 7,26 \\
\hline Berkhout & $-0,0317$ & $-0,19$ & 1,6701 & 10,13 & 1,6704 & 10,14 \\
\hline Curtis (1967) & $-0,0022$ & $-0,01$ & 1,1759 & 7,13 & 1,1759 & 7,13 \\
\hline Exponencial (1) & $-0,0019$ & $-0,01$ & 1,1591 & 7,03 & 1,1591 & 7,03 \\
\hline Exponencial (2) & 0,0664 & 0,40 & 1,6661 & 10,11 & 1,6674 & 10,12 \\
\hline Exponencial (3) & $-0,0394$ & $-0,24$ & 1,9817 & 12,02 & 1,9821 & 12,03 \\
\hline Exponencial (4) & $-0,0022$ & $-0,01$ & 1,1759 & 7,13 & 1,1759 & 7,13 \\
\hline Finger (1992) & $4,04382 \mathrm{E}-15$ & $2,45 \mathrm{E}-14$ & 1,6244 & 9,86 & 1,6244 & 9,86 \\
\hline Henricksen (1950) & $-2,0062 E-14$ & $-1,22 \mathrm{E}-13$ & 1,2150 & 7,37 & 1,2150 & 7,37 \\
\hline Hiperbólico (1) & $-2,5789 \mathrm{E}-12$ & $-1,56 \mathrm{E}-11$ & 1,6204 & 9,83 & 1,6204 & 9,83 \\
\hline Linear Simples & $8,26006 \mathrm{E}-15$ & $5,01 \mathrm{E}-14$ & 1,4493 & 8,79 & 1,4493 & 8,79 \\
\hline Não Referenciado (1) & 0,0684 & 0,42 & 1,1894 & 7,22 & 1,1913 & 7,23 \\
\hline Naslung (1) & 1,1010 & 6,68 & 1,3145 & 7,98 & 1,7147 & 10,40 \\
\hline Naslung (2) & 0,0838 & 0,51 & 1,1928 & 7,24 & 1,1957 & 7,26 \\
\hline Naslung (Prodan) (1) & 1,1030 & 6,69 & 1,2984 & 7,88 & 1,7037 & 10,34 \\
\hline Naslung (Prodan) (2) & 0,0850 & 0,52 & 1,1673 & 7,08 & 1,1704 & 7,10 \\
\hline Parabólico (1) & $1,02036 \mathrm{E}-14$ & $6,19 \mathrm{E}-14$ & 1,1713 & 7,11 & 1,1713 & 7,11 \\
\hline Parabólico (2) & $9,24646 \mathrm{E}-13$ & $5,61 \mathrm{E}-12$ & 1,1599 & 7,04 & 1,1599 & 7,04 \\
\hline Parabólico (3) & $-0,0100$ & $-0,06$ & 1,2199 & 7,40 & 1,2200 & 7,40 \\
\hline Petterson (1) & 0,0674 & 0,41 & 1,1867 & 7,20 & 1,1886 & 7,21 \\
\hline Petterson (2) & 0,0684 & 0,42 & 1,1894 & 7,22 & 1,1913 & 7,23 \\
\hline Pollanschutz & 0,0551 & 0,33 & 1,1749 & 7,13 & 1,1762 & 7,14 \\
\hline Potencial (1) & $-0,0179$ & $-0,11$ & 1,3098 & 7,95 & 1,3099 & 7,95 \\
\hline Potencial (2) & 0,0439 & 0,27 & 1,3417 & 8,14 & 1,3424 & 8,15 \\
\hline Potencial (3) & 0,0439 & 0,27 & 1,3417 & 8,14 & 1,3424 & 8,15 \\
\hline Prodan (1965) (1) & 0,0850 & 0,52 & 1,1673 & 7,08 & 1,1704 & 7,10 \\
\hline Prodan (1965) (2) & 0,0933 & 0,57 & 1,1626 & 7,05 & 1,1663 & 7,08 \\
\hline Sanquetta et al, (2013) & $-3,1786 \mathrm{E}-13$ & $-1,93 \mathrm{E}-12$ & 1,2138 & 7,36 & 1,2138 & 7,36 \\
\hline Schneider (1986) (1) & $3,36985 \mathrm{E}-15$ & $2,04 \mathrm{E}-14$ & 1,2642 & 7,67 & 1,2642 & 7,67 \\
\hline Schneider (1986) (2) & $1,08306 \mathrm{E}-14$ & $6,57 \mathrm{E}-14$ & 1,4493 & 8,79 & 1,4493 & 8,79 \\
\hline Schumacher (1986) (1) & $-4,9372 E-15$ & $-3,00 E-14$ & 1,1713 & 7,11 & 1,1713 & 7,11 \\
\hline Schumacher (1986) (2) & $-0,0179$ & $-0,11$ & 1,3098 & 7,95 & 1,3099 & 7,95 \\
\hline Schumacher-hall (i) & $-0,0179$ & $-0,11$ & 1,3098 & 7,95 & 1,3100 & 7,95 \\
\hline Semilogarítmico (i) & $-3,3900 \mathrm{E}-13$ & $-2,06 \mathrm{E}-12$ & 1,1949 & 7,25 & 1,1949 & 7,25 \\
\hline Silva (1980) & 5,0211 & 30,47 & 1,5169 & 9,20 & 5,2452 & 31,83 \\
\hline Stoffels \& Soest (1953) & $-0,0186$ & $-0,11$ & 1,3435 & 8,15 & 1,3436 & 8,15 \\
\hline Stoffels \& Soest (1953) (2) & 0,0439 & 0,27 & 1,3417 & 8,14 & 1,3424 & 8,15 \\
\hline Trorey (1932) & $1,02036 \mathrm{E}-14$ & $6,19 \mathrm{E}-14$ & 1,1713 & 7,11 & 1,1713 & 7,11 \\
\hline
\end{tabular}

Em que: $\bar{e}$ é o víes absoluto, $\bar{e}_{\%}$ é o viés relativo, $S_{e}$ é a precisão absoluta, $S_{e \%}$ é a precisão relativa, $m_{x}$ é a acurácia absoluta e $m_{x \%}$ é a acurácia relativa. 